PESQUIMAT 24(2): $13-33$ (2021)

ISSN:1560-912X/ ISSN-E:1609-8439

https://doi.org/10.15381/pesquimat.v24i2.21674

Facultad de Ciencias Matemáticas - UNMSM

\title{
Una teoría de cohomología local generalizada
}

\section{Napoleón Caro Tuesta ${ }^{1}$, Sofía Irena Durán Quiñones ${ }^{2}$, Wilfredo Mendoza Quispe ${ }^{3}$}

Resumen: En este trabajo introducimos ciertos funtores de cohomología local que generalizan los estudiados en [9]. Demostramos que sus módulos de cohomología local pueden ser obtenidos como los módulos de cohomología de un complejo de Cech generalizado. También proponemos una noción de homología local. En este contexto probamos que la homología local de un módulo Matlis reflexivo (en el sentido de [2]) se puede expresar como el límite inverso de determinados módulos Tor.

Palabras clave: sistema de ideales, torsión, cohomología local, complejo de Čech, homología local, módulo Matlis reflexivo.

\section{A generalized local cohomology theory}

Abstract: In this work we introduce certain local cohomology functors that generalize those studied in 9 . It is show that their local cohomology modules can be computed as the cohomology modules of a generalized Čech complex. Also we propose a notion of local homology. In this context we prove that the local homology of a Matlis reflexive module (in the sense of [2]) can be expressed as the inverse limit of certain Tor modules.

Keywords: system of ideals, torsion, local cohomology, Čech complex, local homology, Matlis reflexive module.

Recibido: 15/11/2021. Aceptado: 30/11/2021. Publicado online: 30/12/2021.

(C) Los autores. Este artículo es publicado por la Revista PESQUIMAT de la Facultad de Ciencias Matemáticas, Universidad Nacional Mayor de San Marcos. Este es un artículo de acceso abierto, distribuido bajo los términos de la licencia Creative Commons Atribución-No Comercial-Compartir Igual 4.0 Internacional.(http://creativecommons.org/licenses/by-nc-sa/4.0/) que permite el uso no comercial, distribución y reproducción en cualquier medio, siempre que la obra original sea debidamente citada. Para uso comercial, por favor póngase en contacto con revistapesquimat.matematica@unmsm.edu.pe

${ }^{1}$ UFPB, Departamento de Matemática, e-mail: napoleon.caro.tuesta@academico.ufpb.br

${ }^{2}$ UNMSM, Facultad de Ciencias Matemáticas, email: sduranq@unmsm.edu.pe

${ }^{3}$ UNMSM, Facultad de Ciencias Matemáticas, e-mail: wmendozaq@unmsm.edu.pe 


\section{Introducción}

La cohomología local fue introducida por Alexander Grothendieck a principios de los años sesenta en [6] y [5]. Desde entonces, la cohomología local se ha convertido en una herramienta indispensable en geometría algebraica y álgebra conmutativa.

En geometría algebraica, la cohomología local fue introducida como una teoría análoga de la cohomología relativa. Recordemos su definición: Sean $X$ un espacio topológico y $Z$ un subconjunto cerrado de $X$. Para cada haz $\mathscr{F}$ de grupos abelianos sobre $X$, el conjunto

$$
\Gamma_{Z}(X, \mathscr{F}):=\{s \in \mathscr{F}(X) \mid \operatorname{Supp}(s) \subseteq Z\}
$$

es un subgrupo del grupo de secciones globales $\mathscr{F}(X)$. Además, si $\varphi: \mathscr{F} \longrightarrow \mathscr{G}$ es un morfismo de haces de grupos abelianos, la restricción de $\varphi_{X}: \mathscr{F}(X) \longrightarrow \mathscr{G}(X)$ para $\Gamma_{Z}(X, \mathscr{F})$ induce un homomorfismo de grupos

$$
\Gamma_{Z}(X, \varphi): \Gamma_{Z}(X, \mathscr{F}) \longrightarrow \Gamma_{Z}(X, \mathscr{G}) .
$$

Las correspondencias $\mathscr{F} \longmapsto \Gamma_{Z}(X, \mathscr{F})$ y $\varphi \longmapsto \Gamma_{Z}(X, \varphi)$ definen un funtor exacto a la izquierda

$$
\Gamma_{Z}(X,-): S h(X) \longrightarrow A b
$$

de la categoría $S h(X)$ de haces abelianos sobre $X$ en la categoría de grupos abelianos $A b$. Desde que $S h(X)$ posse suficientes objetos inyectivos existen los funtores derivados a la derecha $\left\{\mathscr{R}^{i} \Gamma_{Z}(X,-)\right\}_{i \geq 0}$ del funtor $\Gamma_{Z}(X,-)$. Para cada entero no negativo $i$, el $i$-ésimo funtor de cohomología local de $X$ soportado en $Z$ es definido por

$$
\mathrm{H}_{Z}^{i}(X,-):=\mathscr{R}^{i} \Gamma_{Z}(X,-) .
$$

Notemos que cuando $Z=X$, la cohomología descrita arriba es precisamente la cohomología de haces sobre $X$.

Consideremos el abierto $U=X \backslash Z$, entonces existe una sucesión exacta larga natural

$$
\begin{aligned}
& 0 \rightarrow \Gamma_{Z}(X, \mathscr{F}) \longrightarrow \Gamma(X, \mathscr{F}) \longrightarrow \Gamma\left(U,\left.\mathscr{F}\right|_{U}\right) \stackrel{\partial}{\longrightarrow} \mathrm{H}_{Z}^{1}(X, \mathscr{F}) \longrightarrow \cdots \\
& \longrightarrow \mathrm{H}_{Z}^{i}(X, \mathscr{F}) \longrightarrow \mathrm{H}^{i}(X, \mathscr{F}) \longrightarrow \mathrm{H}^{i}\left(U,\left.\mathscr{F}\right|_{U}\right) \stackrel{\partial}{\rightarrow} \mathrm{H}_{Z}^{i+1}(X, \mathscr{F}) \rightarrow \cdots
\end{aligned}
$$

Esta sucesión muestra que los grupos de cohomología $\mathrm{H}_{Z}^{i}(X, \mathscr{F})$ juegan un rol semejante a los grupos de cohomología relativa de $X$ con respecto a $U$. De hecho, para "buenos espacios" en los cuales la cohomología de haces coincide con la cohomología singular (por ejemplo, espacios de Hausdorff paracompactos), existe un isomorfismo

$$
\mathrm{H}_{Z}^{i}(X, \underline{G}) \cong \mathrm{H}^{i}(X, U, G),
$$

donde $G$ es un grupo Abeliano, $\underline{G}$ es el haz constante asociado a $G$ y $\mathrm{H}^{i}(X, U, G)$ es el $i$-ésimo grupo de cohomología singular de $X$ relativo a $U$ con coeficientes en $G$.

Por otro lado, si $V \subseteq X$ es un conjunto abierto tal que $Z \subseteq V$, entonces existe un isomorfismo de $\delta$-funtores

$$
\mathrm{H}_{Z}^{i}(X, \mathscr{F}) \cong \mathrm{H}_{Z}^{i}\left(V,\left.\mathscr{F}\right|_{V}\right)
$$

Tal resultado es un análogo al conocido Teorema de la Excisión de la cohomología singular.

A continuación consideremos un esquema $\left(X, \mathscr{O}_{X}\right), Z \subseteq X$ un subconjunto cerrado y $\mathscr{F}$ un $\mathscr{O}_{X}$-módulo cuasi-coherente. Supongamos que existe un subconjunto abierto afín $V=\operatorname{Spec} A$ de $X$ tal que $Z \subseteq V$. Entonces

$$
\mathrm{H}_{Z}^{i}(X, \mathscr{F}) \cong \mathrm{H}_{Z}^{i}\left(V,\left.\mathscr{F}\right|_{V}\right) \cong \mathrm{H}_{Z}^{i}(V, \widetilde{M}),
$$


donde $M:=\mathscr{F}(V)$ y $\widetilde{M}$ es el haz de $\mathscr{O}_{X}$-módulos asociado a $M$. Pero

$$
\Gamma_{Z}(V, \widetilde{M})=\{m \in M \mid \operatorname{Supp}(m) \subseteq Z\} .
$$

Por lo tanto, cuando $A$ es un anillo Noetheriano y $Z=V(\mathfrak{a})$ para algún ideal $\mathfrak{a}$ de $A$ obtenemos la siguiente igualdad:

$$
\Gamma_{Z}(V, \widetilde{M})=\left\{m \in M \mid \mathfrak{a}^{n} m=0 \text { para algún } n \in \mathbb{N}\right\} .
$$

La última relación permite abordar la teoría de la cohomología local desde una perspectiva totalmente algebraica. Sean $A$ un anillo conmutativo (no necesariamente Noetheriano) y a un ideal de $A$. Dado un $A$-módulo $M$, el submódulo de a-torsión de $M$ es definido por

$$
\Gamma_{\mathfrak{a}}(M):=\left\{m \in M \mid \mathfrak{a}^{n} m=0 \text { para algún } n \in \mathbb{N}\right\} .
$$

Si $f: M \longrightarrow N$ es un homomorfismo $A$-lineal, su restricción a $\Gamma_{\mathfrak{a}}(M)$ induce un homomorfismo $\Gamma_{\mathfrak{a}}(f): \Gamma_{\mathfrak{a}}(M) \longrightarrow \Gamma_{\mathfrak{a}}(N)$. Las correspondencias $M \longmapsto \Gamma_{\mathfrak{a}}(M)$ y $f \longmapsto \Gamma_{\mathfrak{a}}(f)$ definen un funtor aditivo exacto a la izquierda

$$
\Gamma_{\mathfrak{a}}(-): \operatorname{Mod}_{A} \longrightarrow \operatorname{Mod}_{A}
$$

de la categoría de $A$-módulos $\operatorname{Mod}_{A}$ en sí misma. Como $\operatorname{Mod}_{A}$ es una categoría con suficientes objetos inyectivos, existen los funtores derivados a la derecha $\left\{\mathscr{R}^{i} \Gamma_{\mathfrak{a}}(-)\right\}_{i \geq 0}$ del funtor $\Gamma_{\mathfrak{a}}(-)$. El $i$-ésimo funtor de cohomología local soportado en $\mathfrak{a}$ es definido por

$$
\mathrm{H}_{\mathfrak{a}}^{i}(-):=\mathscr{R}^{i} \Gamma_{\mathfrak{a}}(-) .
$$

Uno de los resultados clásicos garantiza la existencia de un isomorfismo natural

$$
\mathrm{H}_{\mathfrak{a}}^{i}(-) \cong \underset{n \in \mathbb{N}}{\lim _{\vec{N}}} \operatorname{Ext}_{A}^{i}\left(A / \mathfrak{a}^{n},-\right) .
$$

Otra aproximación interesante que permite calcular la cohomología local soportada en un ideal es la siguiente: Dado un elemento $a$ del anillo $A$ consideremos el complejo de $A$-módulos

$$
\check{\mathrm{C}}_{a}: \quad 0 \longrightarrow A \stackrel{\iota}{\longrightarrow} A_{a} \longrightarrow 0,
$$

donde $A$ es colocado en la posición $0, A_{a}$ en la posición 1 y $\iota: A \longrightarrow A_{a}$ es el homomorfismo de localización. Para una sucesión finita $\bar{a}=a_{1}, a_{2}, \ldots, a_{n}$ de elementos de $A$, el complejo de Čech algebraico es definido por

$$
\check{\mathrm{C}}_{\bar{a}}:=\check{\mathrm{C}}_{a_{1}} \otimes \check{\mathrm{C}}_{a_{2}} \otimes \cdots \otimes \check{\mathrm{C}}_{a_{n}} .
$$

Supongamos que $\mathfrak{a}=\left(a_{1}, \ldots, a_{n}\right)$ es un ideal finitamente generado de $A$, entonces existe un isomorfismo natural

$$
\mathrm{H}_{\mathfrak{a}}^{i}(-) \cong \mathrm{H}^{i}\left(\check{\mathrm{C}}_{\bar{a}} \otimes-\right) .
$$

La teoría de cohomología local en su contexto algebraico ha sido ampliamente estudiada y es una área fecunda de investigación. De hecho existen muchos artículos y libros en esta dirección. Ver por ejemplo el excelente libro de T.Y. Brodman and R. Sharp ([3]), así como las referencias que aparecen en el texto.

Ahora recordemos que una familia de soportes en un espacio topológico $X$ es una colección $\Omega$ formada por subconjuntos cerrados de $X$ tales que

(1) Si $S_{1}, S_{2} \in \Omega$, entonces $S_{1} \cup S_{2} \in \Omega$;

(2) Si $S_{1} \in \Omega$ y $S_{2} \subseteq S_{1}$ es un subconjunto cerrado de $X$, entonces $S_{2} \in \Omega$. 
Familias de soportes fueron introducidas para estudiar una variante de la cohomología de haces, la denominada cohomología con soportes en $\Omega$.

En el contexto algebraico existe una noción análoga a la de familia de soportes, a saber: Un sistema completo de ideales es un conjunto $\Phi$ de ideales de un anillo conmutativo $A$ tal que

(i) Si $\mathfrak{a}_{1}, \mathfrak{a}_{2} \in \Phi$, entonces $\mathfrak{a}_{1} \cdot \mathfrak{a}_{2} \in \Phi$;

(ii) Si $\mathfrak{a}_{1} \in \Phi$ y $\mathfrak{a}_{2} \supseteq \mathfrak{a}_{1}$ es un ideal de $A$, entonces $\mathfrak{a}_{2} \in \Phi$.

Tal noción fue introducida por T.Y. Brodman and R. Sharp en [3], y por K. Divanni-Azar, R. Naghipour and M.Tousi en [4, donde los autores presentan brevemente los módulos de cohomología local generalizada soportada en $\Phi$,

$$
\mathrm{H}_{\Phi}^{i}(M):=\mathscr{R}^{i} \Gamma_{\Phi}(M) .
$$

Aquí $\Gamma_{\Phi}(-)$ es el funtor (exacto a la izquierda) de la categoría de $A$-módulos sobre sí misma que asocia a cada $A$-módulo $M$ el $A$-módulo,

$$
\Gamma_{\Phi}(M)=\{x \in M \mid \mathfrak{a} x=0 \text { para algún } \mathfrak{a} \in \Phi\} .
$$

Los fundamentos de esta teoría de cohomología local generalizada, así como versiones generalizadas de resultados clásicos, son estudiados con mayor profundidad por L. A. Alba-Sarria, R. Callejas-Bedregal y N. Caro-Tuesta en [1]. Como nuestro trabajo está soportado sobre tal teoría, recopilamos en la sección 2 algunas nociones básicas de dicha teoría. Para beneficio del lector, incluimos demostraciones detalladas de cada una de las proposiciones enunciadas.

En [9], R. Takahashi, Y. Yoshini and Y. Yoshisawa introdujeron la llamada cohomología local con respecto a un par de ideales $\mathfrak{a} y \mathfrak{b}$ de $A$ como una generalización de la teoría de cohomología local para un ideal. Más precisamente, a cada $A$-módulo $M$ asociaron el conjunto

$$
\Gamma_{\mathfrak{a}, \mathfrak{b}}(M):=\left\{x \in M \mid \mathfrak{a}^{n} x \subseteq \mathfrak{b} x \text { para algún entero positivo } n\right\} .
$$

Como es de esperar, tal correspondencia define un funtor exacto a la izquierda

$$
\Gamma_{\mathfrak{a}, \mathfrak{b}}(-): \operatorname{Mod}_{A} \longrightarrow \operatorname{Mod}_{A},
$$

cuyos funtores derivados a la derecha,

$$
\mathrm{H}_{\mathfrak{a}, \mathfrak{b}}^{i}(M):=\mathscr{R}^{i} \Gamma_{\mathfrak{a}, \mathfrak{b}}(M),
$$

son los módulos de cohomología local con respecto al par de ideales $\mathfrak{a}$ y $\mathfrak{b}$. Uno de los aspectos importantes de esta teoría es que módulos de cohomología local con respecto a un par de ideales pueden ser calculados como los módulos de cohomología de un complejo generalizado de Čech. De manera más explícita, para un elemento $a \in A$, los autores consideran el conjunto multiplicativo $S_{a, \mathfrak{b}}:=\left\{a^{n}+b \mid n \in \mathbb{N}, b \in \mathfrak{b}\right\}$ y definen el complejo de $A$-módulos

$$
\check{\mathrm{C}}_{a, \mathfrak{b}}: \quad 0 \longrightarrow A \stackrel{\iota}{\longrightarrow} A_{a, \mathfrak{b}} \longrightarrow 0
$$

donde $A$ es colocado en la posición 0 , el módulo de fracciones $A_{a, \mathfrak{b}}:=S_{a, \mathfrak{b}}^{-1} A$ está en la posición $1 \mathrm{y} \iota: A \longrightarrow A_{a, \mathfrak{b}}$ es el mapa de localización. Para una sucesión finita $\bar{a}=a_{1}, a_{2}, \ldots, a_{n}$ de elementos de $A$, definen el complejo de Čech con respecto a la sucesión a y el ideal $\mathfrak{b}$ por

$$
\check{\mathrm{C}}_{\bar{a}, \mathfrak{b}}:=\check{\mathrm{C}}_{a_{1}, \mathfrak{b}} \otimes \check{\mathrm{C}}_{a_{2}, \mathfrak{b}} \otimes \cdots \otimes \check{\mathrm{C}}_{a_{n}, \mathfrak{b}} .
$$

Y demuestran que si $\mathfrak{a}=\left(a_{1}, \ldots, a_{n}\right)$ es el ideal generado por los elementos $a_{1}, . ., a_{n}$, entonces existe un isomorfismo natural

$$
\mathrm{H}_{\mathfrak{a}, \mathfrak{b}}^{i}(-) \cong \mathrm{H}^{i}\left(\check{\mathrm{C}}_{\bar{a}, \mathfrak{b}} \otimes-\right) .
$$


Como una generalización de los funtores estudiados en [9], introducimos en la sección 3 los funtores de cohomología local con respecto a un ideal y un conjunto de ideales de A. La principal diferencia radica en que el estudio de nuestra teoría local es abordado en el contexto de la cohomología generalizada descrita en la sección 2. De manera más explícita, dados un ideal $\mathfrak{a} \unlhd A$ y un conjunto no vacío $\varphi$ de ideales de $A$, definimos el funtor

$$
\Gamma_{\mathfrak{a}, \varphi}(-):=\Gamma_{\mathcal{G}(\mathfrak{a}, \varphi)}(-): \operatorname{Mod}_{A} \longrightarrow \operatorname{Mod}_{A},
$$

donde $\mathcal{G}(\mathfrak{a}, \varphi)$ es un sistema completo de ideales de $A$ descrito en la subsección 3.1. La importancia este tipo especial de cohomología local radica en que puede ser calculada como la cohomología de cierto complejo de $A$-módulos. De forma más concreta, para un elemento $a \in A$, primero consideramos el conjunto multiplicativo

$$
S_{a, \varphi}:=\left\{a^{n}+b \mid n \geq 0 \text { y } b \in \mathfrak{b} \text { para algún } \mathfrak{b} \in\langle\varphi\rangle\right\} .
$$

Luego definimos el complejo de $A$-módulos

$$
\mathrm{C}_{\mathfrak{a}, \varphi}^{\bullet}: \quad 0 \longrightarrow A \stackrel{\iota}{\longrightarrow} A_{a, \mathfrak{b}} \longrightarrow 0,
$$

donde $A$ es colocado en la posición 0 , el módulo de fracciones $A_{a, \varphi}:=S_{a, \varphi}^{-1} A$ está en la posición 1 y $\iota: A \longrightarrow A_{a, \mathfrak{b}}$ es el mapa de localización. Para una sucesión finita $\bar{a}=a_{1}, a_{2}, \ldots, a_{n}$ de elementos de $A$, definimos el complejo de Čech con respecto a la sucesión $\bar{a}$ y el conjunto de ideales $\varphi$ como

$$
C_{\bar{a}, \varphi}^{\bullet}:=\check{\mathrm{C}}_{a_{1}, \varphi} \otimes \check{\mathrm{C}}_{a_{2}, \varphi} \otimes \cdots \otimes \check{\mathrm{C}}_{a_{n}, \varphi} \text {. }
$$

Y entonces demostramos en el Teorema 3.2.1 que si $\mathfrak{a}=\left(a_{1}, \ldots, a_{n}\right)$ es el ideal generado por los elementos $a_{1}, . ., a_{n}$, entonces existe un isomorfismo natural

$$
\mathrm{H}_{\mathfrak{a}, \varphi}^{i}(-) \cong \mathrm{H}^{i}\left(\mathrm{C}_{\bar{a}, \varphi}^{\bullet} \otimes-\right) .
$$

Finalmente, en la subsección 3.3 introducimos el co-complejo de Cech de un módulo $M$ con respecto a la sucesión a y a un conjunto de ideales $\varphi$ como el complejo de $A$-módulos $\operatorname{Hom}\left(\mathcal{C}_{\bar{a}, \varphi}^{\bullet}, M\right)$. Luego definimos el $i$-ésimo funtor de homología local com respecto al par $(\mathfrak{a}, \varphi)$ por

$$
\mathrm{H}_{i}^{\mathfrak{a}, \varphi}(-):=\mathrm{H}_{i}\left(\operatorname{Hom}\left(\mathcal{C}_{\bar{a}, \varphi}^{\bullet},-\right)\right)
$$

como noción dual de la cohomología local. Y entonces demostramos en el Teorema 3.3.1 que si $M$ es un $A$-módulo Matlis reflexivo (en el sentido de [2]), existe un isomorfismo natural

$$
\mathrm{H}_{i}^{\mathfrak{a}, \varphi}(M) \cong \lim _{\mathfrak{b} \in \overleftarrow{\mathcal{G}(\mathfrak{a}, \varphi)}} \operatorname{Tor}_{i}^{A}(A / \mathfrak{b}, M)
$$

\section{Cohomología local con respecto a un sistema de ideales}

A lo largo de esta sección, $A$ denotará un anillo conmutativo con elemento unitario. Escribiremos $\mathfrak{a} \unlhd A$ para indicar que $\mathfrak{a}$ es un ideal de $A$.

\subsection{Sistemas de ideales}

Definición 2.1.1. Sea $\Phi$ un conjunto no vacío de ideales de $A$. Decimos que $\Phi$ es un sistema de ideales de $A$ si, para cada par $\mathfrak{a}, \mathfrak{b} \in \Phi$ existe $\mathfrak{c} \in \Phi$ tal que $\mathfrak{c} \subseteq \mathfrak{a} \mathfrak{b}$.

Un sistema de ideales es completo cuando satisface la siguiente condición: si $\mathfrak{a} \in \Phi$ y $\mathfrak{b}$ es un ideal de $A$ tal que $\mathfrak{b} \supseteq \mathfrak{a}$, entonces $\mathfrak{b} \in \Phi$. 
Ejemplo 2.1.1. Un ejemplo importante de sistema de ideales de $A$ es el conjunto formado por todas las potencias de un ideal a de $A$, i.e.,

$$
\left\{\mathfrak{a}^{n} \mid n \geq 0\right\} .
$$

Ejemplo 2.1.2. Dada una colección no vacía $\varphi$ de ideales de $A$, el conjunto

$$
\langle\varphi\rangle:=\left\{\mathfrak{b} \unlhd A \mid \mathfrak{b} \supseteq \mathfrak{a}_{1} \cdots \mathfrak{a}_{n} \quad \text { para algunos } \mathfrak{a}_{1}, \ldots, \mathfrak{a}_{n} \in \varphi\right\}
$$

es un sistema de ideales completo, que llamaremos sistema generado por $\varphi$. De hecho, $\langle\varphi\rangle$ es el menor (con respecto a la inclusión) sistema de ideales completo que contiene a $\varphi$. Cuando $\varphi$ consiste de un único ideal $\mathfrak{a}$ de $A$, el sistema generado por $\varphi$ será denotado por $\langle\mathfrak{a}\rangle$. De esa manera,

$$
\langle\mathfrak{a}\rangle=\left\{\mathfrak{b} \unlhd A \mid \mathfrak{b} \supseteq \mathfrak{a}^{n} \text { para algún entero } n \geq 1\right\} .
$$

Ejemplo 2.1.3. Sean $f: A \longrightarrow B$ un homomorfismo de anillos y $\Phi$ un sistema completo de ideales de $A$, entonces

$$
f_{*}(\Phi):=\left\{\mathfrak{b} \unlhd B \mid f^{-1}(\mathfrak{b}) \in \Phi\right\}
$$

es un sistema completo de ideales de $B$. Más aún,

$$
f_{*}(\Phi)=\langle\{\mathfrak{a} B \mid \mathfrak{a} \in \Phi\}\rangle .
$$

Por otro lado, si $\Psi$ es un sistema completo de ideales de $B$, entonces

$$
f^{*}(\Psi):=\left\{\mathfrak{a} \unlhd A \mid \mathfrak{a} \in\left\langle f^{-1}(\mathfrak{b})\right\rangle \text { para algún ideal } \mathfrak{b} \in \Psi\right\}
$$

es un sistema completo de ideales de $A$.

Recordemos que el "conjunto de ceros" de un ideal a de $A$ es dado por

$$
\mathrm{V}(\mathfrak{a})=\{\mathfrak{p} \in \operatorname{Spec} A: \mathfrak{p} \supseteq \mathfrak{a}\}
$$

Lema 2.1.1. Supongamos que A es Noetheriano y sea $\Phi$ una colección no vacía de ideales de A. Entonces $\Phi$ es un sistema completo, si y solo si, posee la siguiente propiedad: un ideal $\mathfrak{a}$ de $A$ es un elemento de $\Phi$, si y solo si, $\mathrm{V}(\mathfrak{a}) \subseteq \Phi$.

Prueba. Supongamos que $\Phi$ es un sistema completo y sea $\mathfrak{a}$ un ideal de $A$ tal que $\mathrm{V}(\mathfrak{a}) \subseteq \Phi$. Desde que $A$ es Noetheriano, el conjunto de ideales primos minimales sobre $\mathfrak{a}$ es finito. Denotemos por $\mathfrak{p}_{1}, \ldots, \mathfrak{p}_{s}$ tales ideales, entonces $\sqrt{\mathfrak{a}}=\mathfrak{p}_{1} \cap \cdots \cap \mathfrak{p}_{s}$. Nuevamente, como $A$ es Noetheriano, existe un entero positivo $r$ tal que $\mathfrak{a} \supseteq \sqrt{\mathfrak{a}}^{r}$. Luego,

$$
\mathfrak{a} \supseteq\left(\mathfrak{p}_{1} \cap \cdots \cap \mathfrak{p}_{s}\right)^{r} \supseteq \mathfrak{p}_{1}^{r} \cdots \mathfrak{p}_{s}^{r} .
$$

Ésto implica que $\mathfrak{a} \in \Phi$. La otra dirección es obvia.

Recíprocamente, supongamos que $\Phi$ tiene la propiedad mencionada. Si $\mathfrak{a} \in \Phi$ y $\mathfrak{b}$ es un ideal de $A$ que contiene $\mathfrak{a}$, la inclusión $V(\mathfrak{b}) \subseteq V(\mathfrak{a})$ implica que $\mathfrak{b} \in \Phi$. Más aún, desde que $V(\mathfrak{a} \mathfrak{b})=V(\mathfrak{a}) \cup V(\mathfrak{b})$ para todo par de ideales $\mathfrak{a}, \mathfrak{b}$ de $A$, obtenemos que $\Phi$ es cerrado por producto de ideales. Por lo tanto $\Phi$ es un sistema completo de ideales.

\subsection{El funtor $\Phi$-torsión}

Sean $\Phi$ un sistema de ideales de $A$ y $M$ un $A$-módulo. Consideremos el conjunto

$$
\Gamma_{\Phi}(M):=\{x \in M \mid \mathfrak{a} x=0 \text { para algún } \mathfrak{a} \in \Phi\} .
$$

Es claro que $0 \in \Gamma_{\Phi}(M)$. Afirmamos que $\Gamma_{\Phi}(M)$ es un submódulo de $M$. En efecto: si $x, y \in$ $\Gamma_{\Phi}(M)$, existen $\mathfrak{a}, \mathfrak{b} \in \Phi$ tales que $\mathfrak{a} x=0$ y $\mathfrak{b} y=0$. Elijamos $\mathfrak{c} \in \Phi$ de modo que $\mathfrak{c} \subseteq \mathfrak{a} \mathfrak{b}$, entonces $\mathfrak{c}(x-y)=0$. Además, $\mathfrak{a}(r x)=r(\mathfrak{a} x)=0$ para todo $r \in A$. 
Lema 2.2.1. Asuma que $A$ es un anillo Noetheriano y que $\Phi$ es un sistema completo de ideales de A. Para todo A-módulo $M$ vale la siguiente igualdad:

$$
\Gamma_{\Phi}(M)=\{x \in M \mid \operatorname{Supp}(A x) \subseteq \Phi\} .
$$

Prueba. Sea $x$ un elemento de $M$. Supongamos que $x \in \Gamma_{\Phi}(M)$, entonces existe $\mathfrak{a} \in \Phi$ tal que $\mathfrak{a} x=0$, esto implica que $\mathfrak{a} \subseteq \operatorname{Ann}(x)$. Como $\Phi$ es completo, $\operatorname{Ann}(x) \in \Phi$. Usando el Lema 2.1.1 obtenemos que $\operatorname{Supp}(A x)=V(\operatorname{Ann}(x)) \subseteq \Phi$.

Recíprocamente, si $\operatorname{Supp}(A x) \subseteq \Phi$, o equivalentemente, $V(\operatorname{Ann}(x)) \subseteq \Phi$. Luego, $\operatorname{Ann}(x) \in \Phi$ por el Lema 2.1.1. Como $\operatorname{Ann}(x) x=0$, concluimos que $x \in \Gamma_{\Phi}(M)$.

Ahora sea $f: M \longrightarrow N$ un homomorfismo de $A$-módulos. Si $x \in M$ es tal que $\mathfrak{a} x=0$ para algún $\mathfrak{a} \in \Phi$, entonces $\mathfrak{a} f(x)=0$. En consecuencia, $f\left(\Gamma_{\Phi}(M)\right) \subseteq \Gamma_{\Phi}(N)$. Por lo tanto, la restricción de $f$ para $\Gamma_{\Phi}(M)$ induce un homomorfismo de $A$-módulos

$$
\Gamma_{\Phi}(f): \Gamma_{\Phi}(M) \longrightarrow \Gamma_{\Phi}(N) .
$$

No es difícil ver que las correspondencias $M \longmapsto \Gamma_{\Phi}(M)$ y $f \longmapsto \Gamma_{\Phi}(f)$ definen un funtor $A$-lineal

$$
\Gamma_{\Phi}(-): \operatorname{Mod}_{A} \longrightarrow \operatorname{Mod}_{A}
$$

de la categoría de $A$-módulos $\operatorname{Mod}_{A}$ en sí misma.

Definición 2.2.1. El funtor $\Gamma_{\Phi}(-)$ es llamado $\Phi$-torsión. Un $A$-módulo $M$ es denominado $\Phi$ torsión cuando $\Gamma_{\Phi}(M)=M$. En el caso que $\Gamma_{\Phi}(M)=0$, decimos que $M$ es libre de $\Phi$-torsión.

Ejemplo 2.2.1. Asuma que $A$ es un anillo Noetheriano y que $\Phi$ es un sistema completo de ideales de $A$. Dado un ideal primo $\mathfrak{p} \in \operatorname{Spec}(A)$, entonces $A / \mathfrak{p}$ es $\Phi$-torsión, si y solamente si, $\mathfrak{p} \in \Phi$. Por otro lado, $A / \mathfrak{p}$ es libre de torsión, si y solamente si, $\mathfrak{p} \notin \Phi$.

Proposición 2.2.1. Para todo sistema $\Phi$ de ideales de $A$ y todo A-módulo $M$, tenemos que

$$
\Gamma_{\Phi}(M)=\bigcup_{\mathfrak{a} \in \Phi} \Gamma_{\mathfrak{a}}(M)
$$

Prueba. Si $x \in \Gamma_{\Phi}(M)$, entonces $\mathfrak{b} x=0$ para algún $\mathfrak{b} \in \Phi$. Por lo tanto, $x \in \Gamma_{\mathfrak{b}}(M)$. Por otro lado, si $x \in \Gamma_{\mathfrak{b}}(M)$ para algún $\mathfrak{b} \in \Phi$, entonces existe $n \geq 1$ tal que $\mathfrak{b}^{n} x=0$. Como $\Phi$ es un sistema de ideales,existe $\mathfrak{c} \in \Phi$ tal que $\mathfrak{c} \subseteq \mathfrak{b}^{n}$. Esto implica que $\mathfrak{c} x=0$ y por lo tanto, $x \in \Gamma_{\Phi}(M)$. En consecuencia, $\Gamma_{\Phi}(M)=\bigcup_{\mathfrak{a} \in \Phi} \Gamma_{\mathfrak{a}}(M)$.

Observación 2.2.1. Equipemos el conjunto $\Phi$ el orden parcial $\preceq$ definido por la inclusión inversa, i.e.,

$$
\mathfrak{a} \preceq \mathfrak{b}, \quad \text { si } \quad \mathfrak{b} \subseteq \mathfrak{a} .
$$

Para un $A$-módulo $M$, las inclusiones $\quad \Gamma_{\mathfrak{a}}(M) \longleftrightarrow \Gamma_{\mathfrak{b}}(M), \quad$ cuando $\mathfrak{a} \preceq \mathfrak{b}$ inducen un sistema directo de $A$-módulos tal que

$$
\underset{\mathfrak{a} \in \Phi}{\lim _{\vec{a}}} \Gamma_{\mathfrak{a}}(M)=\bigcup_{\mathfrak{a} \in \Phi} \Gamma_{\mathfrak{a}}(M) .
$$

Por lo tanto, la Proposición 2.2.1 puede ser reescrita de la siguiente manera: Para todo sistema $\Phi$ de ideales de $A$ y todo $A$-módulo $M$, tenemos que

$$
\Gamma_{\Phi}(M)=\underset{\mathfrak{a} \in \Phi}{\lim _{\mathfrak{a}}} \Gamma_{\mathfrak{a}}(M) .
$$


Lema 2.2.2. El funtor $\Gamma_{\Phi}(-)$ es exacto a la izquierda.

Prueba. Sea

$$
0 \longrightarrow M \stackrel{f}{\longrightarrow} N \stackrel{g}{\longrightarrow} L \longrightarrow 0
$$

una sucesión exacta de $A$-módulos. Desde que $\Gamma_{\Phi}(-)$ es un funtor tenemos que $\Gamma_{\Phi}(g) \circ \Gamma_{\Phi}(f)=$ $\Gamma_{\Phi}(g \circ f)=\Gamma_{\Phi}(0)=0$. Por otro lado, si $y \in \operatorname{Ker}\left(\Gamma_{\Phi}(g)\right)$, entonces existe $\mathfrak{a} \in \Phi$ tal que $\mathfrak{a} y=0$ y $g(y)=0$. Como $\operatorname{Ker}(g)=\operatorname{Im}(f)$ podemos escribir $y=f(x)$ para algún $x \in M$. En vista que $f$ es un homomorfismo $A$-lineal inyectivo, $\operatorname{Ann}(x)=\operatorname{Ann}(f(x))=\operatorname{Ann}(y)$. Esta igualdad implica que $\mathfrak{a} \subseteq \operatorname{Ann}(x)$ y por lo tanto, $x \in \Gamma_{\Phi}(M)$. De esa manera, $\operatorname{Ker}\left(\Gamma_{\Phi}(g)\right)=\operatorname{Im}\left(\Gamma_{\Phi}(f)\right)$. Finalmente, si $x \in \operatorname{Ker}\left(\Gamma_{\Phi}(f)\right)$, la igualdad $\operatorname{Ann}(x)=\operatorname{Ann}(f(x))$ garantiza que $x=0$. En conclusión, la sucesión

$$
0 \longrightarrow \Gamma_{\Phi}(M) \stackrel{\Gamma_{\Phi}(f)}{\longrightarrow} \Gamma_{\Phi}(N) \stackrel{\Gamma_{\Phi}(g)}{\longrightarrow} \Gamma_{\Phi}(L)
$$

es exacta.

Observación 2.2.2. En general el funtor $\Gamma_{\Phi}(-)$ no es exacto a la derecha. Por ejemplo, consideremos $A=\mathbb{Z}$ el anillo de los números enteros y $\Phi:=\langle\{(2),(3),(5), \ldots\}\rangle$, el sistema generado por todos los ideales maximales de $\mathbb{Z}$. Entonces $\Gamma_{\Phi}(\mathbb{Z})=0$. En efecto, si $x \in \Gamma_{\Phi}(\mathbb{Z})$, entonces existe $\mathfrak{a} \in \Phi$ tal que $\mathfrak{a} x=0$. Por definición de sistema generado, $\mathfrak{a} \supseteq\left(\mathfrak{p}_{1}\right) \cdots\left(\mathfrak{p}_{s}\right)$ para algunos números primos $p_{1}, \ldots, p_{s}$. En particular, $p_{1} \cdots p_{s} x=0$, lo que implica que $x=0$.

Ahora fijemos un número primo $p$. Afirmamos que $\Gamma_{\Phi}(\mathbb{Z} / p \mathbb{Z})=\mathbb{Z} / p \mathbb{Z}$. En efecto, desde que $\Gamma_{(q)}(\mathbb{Z} / p \mathbb{Z})=0$ cuando $q \neq p$ y $\Gamma_{(p)}(\mathbb{Z} / p \mathbb{Z})=\mathbb{Z} / p \mathbb{Z}$, la igualdad

$$
\Gamma_{\Phi}(\mathbb{Z} / p \mathbb{Z})=\bigcup_{q \in \operatorname{Spec}(\mathbb{Z})} \Gamma_{(q)}(\mathbb{Z} / p \mathbb{Z})
$$

demuestra la afirmación (ver el Lema 2.2.1).

A continuación consideremos la sucesión exacta

$$
0 \longrightarrow \mathbb{Z} \stackrel{p}{\longrightarrow} \mathbb{Z} \longrightarrow \mathbb{Z} / p \mathbb{Z} \longrightarrow 0 .
$$

Después de aplicar el funtor $\Phi$-torsión obtenemos la sucesión

$$
0 \longrightarrow 0 \longrightarrow 0 \longrightarrow \mathbb{Z} / p \mathbb{Z} \longrightarrow 0,
$$

que claramente no es exacta a la derecha.

\subsection{Funtores de cohomología local generalizada}

A lo lago de esta subsección, $\Phi$ denotará un sistema de ideales de $A$.

Definición 2.3.1. Para cada $i \geq 0$, el $i$-ésimo funtor de cohomología local generalizada soportada en $\Phi$ es definido por

$$
\mathrm{H}_{\Phi}^{i}(-):=\mathcal{R}^{i} \Gamma_{\Phi}(-),
$$

donde $\mathcal{R}^{i} \Gamma_{\Phi}(-)$ es el $i$-ésimo funtor derivado a la derecha del funtor $\Phi$-torsion.

De manera explícita, para cada $A$-módulo $M$, el $A$-módulo $H_{\Phi}^{i}(M)$ es obtenido de la siguiente manera: Sea $0 \longrightarrow M \longrightarrow E^{\bullet}$ una resolución inyectiva de $M$, entonces

$$
\mathrm{H}_{\Phi}^{i}(M)=\mathrm{H}^{i}\left(\Gamma_{\Phi}\left(E^{\bullet}\right)\right) .
$$

Siguen directamente de la definición, las siguientes propiedades:

(i) Los funtores $\Gamma_{\Phi}(-)$ y $\mathrm{H}_{\Phi}^{0}(-)$ son naturalmente isomorfos, pues $\Gamma_{\Phi}(-)$ es exacto a la izquierda. 
(ii) Si $I$ es un $A$-módulo inyectivo, entonces $\mathrm{H}_{\Phi}^{i}(I)=0$ para todo $i \geq 1$.

(iii) Toda sucesión exacta corta $0 \longrightarrow L \longrightarrow M \longrightarrow N \longrightarrow 0$ induce naturalmente una sucesión exacta larga en los módulos de cohomología local

$$
0 \longrightarrow \mathrm{H}_{\Phi}^{0}(L) \longrightarrow \mathrm{H}_{\Phi}^{0}(M) \longrightarrow \mathrm{H}_{\Phi}^{0}(N) \stackrel{\delta_{\Phi}^{0}}{\longrightarrow} \mathrm{H}_{\Phi}^{1}(L) \longrightarrow \mathrm{H}_{\Phi}^{1}(M) \longrightarrow \mathrm{H}_{\Phi}^{1}(N) \stackrel{\delta_{\Phi}^{1}}{\longrightarrow} \cdots
$$

Como antes, consideremos el orden parcial $\preceq$ en $\Phi$ definido por la inclusión inversa. Sean $M$ un $A$-módulo y $0 \longrightarrow M \longrightarrow E^{\bullet}$ una resolución inyectiva de $M$. Dado un par de ideales $\mathfrak{a}, \mathfrak{b} \in \Phi$ tal que $\mathfrak{a} \preceq \mathfrak{b}$, las inclusiones $\Gamma_{\mathfrak{a}}\left(E^{i}\right) \longleftrightarrow \Gamma_{\mathfrak{b}}\left(E^{i}\right)$ definen un morfismo de complejos

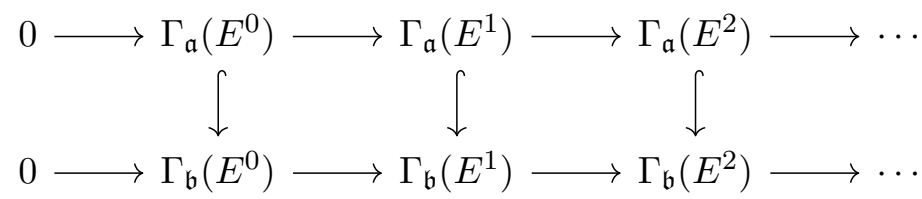

Tal morfismo induce, para cada $i \geq 0$, un homomorfismo de $A$-módulos

$$
\iota_{\mathfrak{b} \mathfrak{a}}^{i}: \mathrm{H}_{\mathfrak{a}}^{i}(M) \longrightarrow \mathrm{H}_{\mathfrak{b}}^{i}(M) .
$$

No es difícil ver que $\left\{\mathrm{H}_{\mathfrak{a}}^{i}(M), \iota_{\mathfrak{b a}}^{i}\right\}$ es un sistema directo de $A$-módulos.

Lema 2.3.1. Para todo sistema completo $\Phi$ de ideales de $A$, la sucesión $\left\{\underset{\mathfrak{a} \in \Phi}{\lim } \mathrm{H}_{\mathfrak{a}}^{i}(-)\right\}_{i \geq 0}$ es un $\delta$-funtor cohomológico.

Prueba. Para $\mathfrak{a} \in \Phi$, cada sucesión exacta corta $A$-módulos

$$
0 \longrightarrow L \longrightarrow M \longrightarrow N \longrightarrow 0
$$

induce una sucesión exacta de módulos

$$
0 \longrightarrow \mathrm{H}_{\mathfrak{a}}^{0}(L) \longrightarrow \mathrm{H}_{\mathfrak{a}}^{0}(M) \longrightarrow \mathrm{H}_{\mathfrak{a}}^{0}(N) \stackrel{\delta_{\mathfrak{a}}^{0}}{\longrightarrow} \mathrm{H}_{\mathfrak{a}}^{1}(L) \longrightarrow \mathrm{H}_{\mathfrak{a}}^{1}(M) \longrightarrow \mathrm{H}_{\mathfrak{a}}^{1}(N) \stackrel{\delta_{\mathfrak{a}}^{1}}{\longrightarrow} \cdots .
$$

Desde que el funtor límite directo es exacto, obtenemos la siguiente sucesión exacta larga

$$
0 \longrightarrow \lim _{\mathfrak{a} \in \Phi} H_{\mathfrak{a}}^{0}(L) \longrightarrow \lim _{\mathfrak{a} \in \Phi} H_{\mathfrak{a}}^{0}(M) \longrightarrow \lim _{\mathfrak{a} \in \Phi} H_{\mathfrak{a}}^{0}(N) \stackrel{\Delta_{\Phi}^{0}}{\longrightarrow} \underset{\mathfrak{a} \in \Phi}{\lim _{\mathfrak{a}}} H_{\mathfrak{a}}^{1}(L) \longrightarrow \cdots,
$$

donde $\Delta_{\Phi}^{i}=\underset{\mathfrak{a} \in \Phi}{\lim } \delta_{\mathfrak{a}}^{i}$ para todo $i \geq 0$.

Por otro lado, dado un diagrama conmutativo con filas exactas

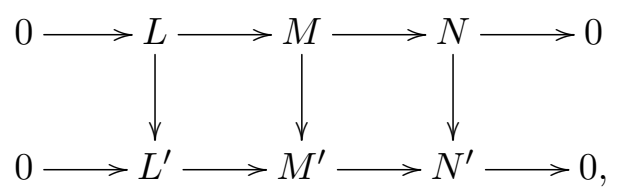

el diagrama

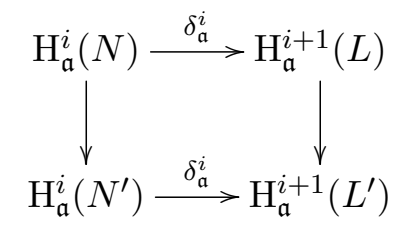


es commutativo para cada ideal $\mathfrak{a}$ en $\Phi$ y para todo $i \geq 0$. Después de tomar límites directos obtenemos que el siguiente diagrama

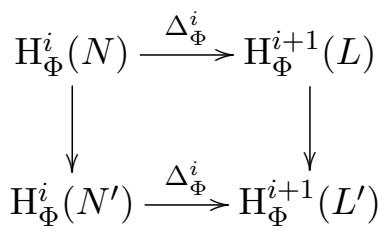

es commutativo, concluyendo que la sucesión $\left\{\underset{\mathfrak{a} \in \Phi}{\lim _{\mathfrak{a}}} H_{\mathfrak{a}}^{i}(-)\right\}_{i \geq 0}$ es un $\delta$-funtor cohomológico.

Proposición 2.3.1. Sea $\Phi$ un sistema de ideales de A.

(i) Existe un único isomorfismo de $\delta$-funtores

$$
\left\{T^{i}: \underset{\mathfrak{a} \in \Phi}{\lim } \mathrm{H}_{\mathfrak{a}}^{i}(-) \longrightarrow \mathrm{H}_{\Phi}^{i}(-)\right\}_{i \geq 0}
$$

tal que $T^{0}=I d$.

(ii) En consecuencia, para todo A-módulo $M$ y todo $i \geq 0$,

$$
\underset{\mathfrak{a} \in \Phi}{\lim _{\mathfrak{a}}} \mathrm{H}_{\mathfrak{a}}^{i}(M) \cong \mathrm{H}_{\Phi}^{i}(M) \text {. }
$$

Prueba. Por la Observación 2.2.1 y el Lema 2.3.1 es suficiente probar que $\underset{\mathfrak{a} \in \Phi}{\lim } H_{\mathfrak{a}}^{i}(I)=0$ para todo $i \geq 1$ y para cualquier $A$-modulo inyectivo 1 . Pero esto sigue directamente del hecho que $\mathrm{H}_{\mathfrak{a}}^{i}(I)=0$ para todo $i \geq 1$ y para todo $\mathfrak{a} \in \Phi$.

Corolario 2.3.1. Para todo $i \geq 0$, existe un isomorfismo natural

$$
\mathrm{H}_{\Phi}^{i}(-) \cong \underset{\mathfrak{a} \in \Phi}{\lim _{\longrightarrow}} \operatorname{Ext}_{A}^{i}(A / \mathfrak{a},-)
$$

Prueba. Como vimos en la Introducción de nuestro trabajo, para cada ideal $\mathfrak{a} \in \Phi$, los funtores $\mathrm{H}_{\mathfrak{a}}^{i}(-)$ y $\underset{n \in \mathbb{N}}{\lim } \operatorname{Ext}_{A}^{i}\left(A / \mathfrak{a}^{n},-\right)$ son naturalmente isomorfos. Por lo tanto, por la Proposición 2.3.1 y desde que límites directos conmutan con límites directos, tenemos los isomorfismos naturales

$$
\mathrm{H}_{\Phi}^{i}(-) \cong \underset{\mathfrak{a} \in \Phi}{\lim _{n \in \mathbb{N}}} \lim _{\vec{n}} \operatorname{Ext}_{A}^{i}\left(A / \mathfrak{a}^{n},-\right) \cong \underset{n \in \mathbb{N}}{\lim _{\mathfrak{a} \in \Phi}} \lim _{\vec{A}} \operatorname{Ext}_{A}^{i}\left(A / \mathfrak{a}^{n},-\right) .
$$

Pero, para cada $n \in \mathbb{N}$ fijo, el conjunto $\left\{\mathfrak{a}^{n}: \mathfrak{a} \in \Phi\right\}$ es un subconjunto cofinal de $\Phi$. En consecuencia,

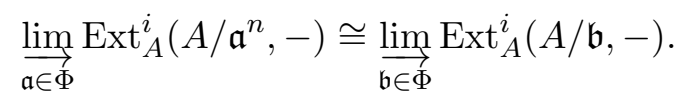

Por lo tanto, tenemos un isomorfismo natural

$$
\mathrm{H}_{\varphi}^{i}(-) \cong \underset{n \in \mathbb{N}}{\lim _{\mathfrak{b} \in \Phi}} \lim _{\longrightarrow} \operatorname{Ext}_{A}^{i}(A / \mathfrak{b},-)=\underset{\mathfrak{b} \in \Phi}{\lim _{\longrightarrow}} \operatorname{Ext}_{A}^{i}(A / \mathfrak{b},-) .
$$

Esto prueba el corolario. 


\section{Los funtores de cohomología $H_{\mathfrak{a}, \varphi}^{i}(-)$}

A lo largo de esta sección, $A$ denotará un anillo conmutativo Noetheriano, a un ideal de $A$ y $\varphi$ un conjunto no vacío de ideales de $A$.

\subsection{El funtor de torsión $\Gamma_{\mathfrak{a}, \varphi}(-)$}

Consideremos el siguiente conjunto de ideales de $A$.

$$
\mathcal{G}(\mathfrak{a}, \varphi):=\{\mathfrak{c} \unlhd A: \mathfrak{b}+\mathfrak{c} \in\langle\mathfrak{a}\rangle \text { para algún ideal } \mathfrak{b} \in\langle\varphi\rangle\}
$$

Explícitamente, un ideal $\mathfrak{c}$ de $A$ es un elemento de $\mathcal{G}(\mathfrak{a}, \varphi)$, si y solo si, existen un número natural $n \geq 1$ y un ideal $\mathfrak{b} \in\langle\varphi\rangle$ tal que $\mathfrak{b}+\mathfrak{c} \supseteq \mathfrak{a}^{n}$.

Lema 3.1.1. El conjunto $\mathcal{G}(\mathfrak{a}, \varphi)$ es un sistema completo de ideales de $A$.

Prueba. $\quad$ i) Sean $\mathfrak{c} \in \mathcal{G}(\mathfrak{a}, \varphi)$ y $\mathfrak{d} \unlhd A$ tal que $\mathfrak{d} \supseteq \mathfrak{c}$. Entonces tenemos las siguientes inclusiones: $\mathfrak{d}+\mathfrak{b} \supseteq \mathfrak{c}+\mathfrak{b} \supseteq \mathfrak{a}^{n}$ para algún $n \geq 1$ y algún $\mathfrak{b} \in\langle\varphi\rangle$. Por lo tanto, $\mathfrak{d} \in \mathcal{G}(\mathfrak{a}, \varphi)$.

ii) Supongamos que $\mathfrak{c}, \mathfrak{d} \in \mathcal{G}(\mathfrak{a}, \varphi)$, entonces podemos escoger números naturales $m$ y $n$ e ideales $\mathfrak{b}, \mathfrak{b}^{\prime} \in\langle\varphi\rangle$ tales que $\mathfrak{c}+\mathfrak{b} \supseteq \mathfrak{a}^{m}$ y $\mathfrak{d}+\mathfrak{b}^{\prime} \supseteq \mathfrak{a}^{n}$. Luego, $\mathfrak{c} \mathfrak{d}+\mathfrak{b} \mathfrak{b}^{\prime} \supseteq(\mathfrak{c}+\mathfrak{b})\left(\mathfrak{d}+\mathfrak{b}^{\prime}\right) \supseteq \mathfrak{a}^{m+n}$. Por i) concluimos que $\mathfrak{c} \mathfrak{d} \in \mathcal{G}(\mathfrak{a}, \varphi)$.

El lema anterior nos permite definir, usando el lenguaje presentado en la sección 2.2, el siguiente funtor.

Definición 3.1.1. El $(\mathfrak{a}, \varphi)$-funtor de torsión $\Gamma_{\mathfrak{a}, \varphi}(-): \operatorname{Mod}_{A} \longrightarrow \operatorname{Mod}_{A}$ es definido por:

$$
\Gamma_{\mathfrak{a}, \varphi}(-):=\Gamma_{\mathcal{G}(\mathfrak{a}, \varphi)}(-) .
$$

De manera más explícita, para cada $A$-módulo $M$ sobre $A$,

$$
\Gamma_{\mathfrak{a}, \varphi}(M)=\{x \in M: \operatorname{Sup}(A x) \subseteq \mathcal{G}(\mathfrak{a}, \varphi)\}
$$

Como $\operatorname{Sup}(A x)=\mathrm{V}(\operatorname{Ann}(x))$, el Lema 2.1.1 implica que un elemento $x$ de $M$ pertenece a $\Gamma_{\mathfrak{a}, \varphi}(M)$ si, y solo sí, $\operatorname{Ann}(x)+\mathfrak{b} \supseteq \mathfrak{a}^{n}$ para algún $n \geq 1$ y algún ideal $\mathfrak{b} \in\langle\varphi\rangle$.

A continuación tomemos un elemento $a \in A$ y consideremos el siguiente conjunto

$$
S_{a, \varphi}=\left\{a^{n}+b: n \geq 0, b \in \mathfrak{b} \text { para algún } \mathfrak{b} \in\langle\varphi\rangle\right\} .
$$

Observamos que $S_{a, \varphi}$ es un subconjunto multiplicativo de $A$. En efecto, es claro que $1 \in S_{a, \varphi}$. Además, si $x, y \in S_{a, \varphi}$ podemos escribir $x=a^{m}+b$ e $y=a^{n}+c$ para algunos enteros no negativos $m, n$ y algunos ideales $\mathfrak{b}, \mathfrak{c} \in \varphi$ con $b \in \mathfrak{b}$ y $c \in \mathfrak{c}$. Por lo tanto, $x y=a^{m+n}+d$, donde $d \in \mathfrak{b}+\mathfrak{c}$. Desde que $\langle\varphi\rangle$ es un sistema completo de ideales, $\mathfrak{b}+\mathfrak{c} \in\langle\varphi\rangle$ y en consecuencia, $x y \in S_{a, \varphi}$.

Dado un $A$-módulo $M$, denotaremos por $M_{a, \varphi}$ el módulo de fracciones de $M$ con respecto al conjunto multiplicativo $S_{a, \varphi}$, i.e., $M_{a, \varphi}=S_{a, \varphi}^{-1} M$.

Lema 3.1.2. Son válidas las siguientes afirmaciones:

i) $0 \in S_{a, \varphi}$ si y solo si $a \in \sqrt{\mathfrak{b}}$ para algún ideal $\mathfrak{b} \in\langle\varphi\rangle$.

ii) Si $\mathfrak{p} \in \mathcal{G}(\mathfrak{a}, \varphi) \cap \operatorname{Spec}(A)$ y $a \in \mathfrak{a}$, entonces $\mathfrak{p} \cap S_{a, \varphi} \neq \emptyset$. 
iii) Si $\mathfrak{a}=\left\langle a_{1}, \ldots ., a_{k}\right\rangle$, entonces la sucesión natural de A-módulos

$$
0 \longrightarrow \Gamma_{\mathfrak{a}, \varphi}(M) \longrightarrow M \longrightarrow \bigoplus_{i=1}^{k} M_{a_{i}, \varphi}
$$

es exacta.

Prueba. i) Supongamos que $0 \in S_{a, \varphi}$, entonces existen un entero no negativo $n$, un ideal $\mathfrak{b} \in \varphi$ y un elemento $b \in \mathfrak{b}$ tal que $0=a^{n}+b$. Esto implica que $a^{n} \in \mathfrak{b}$ o equivalentemente, $a \in \sqrt{\mathfrak{b}}$. Recíprocamente, si $a \in \sqrt{\mathfrak{b}}$ para algún ideal $\mathfrak{b} \in \varphi$, entonces $b:=-a^{n} \in \mathfrak{b}$. Luego podemos escribir $0=a^{n}+b$, lo que muestra que $0 \in S_{a, \varphi}$.

ii) Supongamos que existe un ideal primo $\mathfrak{p}$ tal que $\mathfrak{p} \in \mathcal{G}(\mathfrak{a}, \varphi)$, entonces $\mathfrak{p}+\mathfrak{b} \supseteq \mathfrak{a}^{n}$ para algún número natural $n \geq 1$ y algún ideal $\mathfrak{b} \in \varphi$. Si además suponemos que $a \in \mathfrak{a}$, es posible escribir $a^{n}=x+b$ con $x \in \mathfrak{p}$ y $b \in \mathfrak{b}$. Luego, $x=a^{n}+(-b) \in S_{\mathfrak{a}, \varphi} \cap \mathfrak{p}$.

iii) El primer homomorfismo $A$-lineal en la sucesión (1) es la inclusión natural $\iota$, que obviamente es inyectivo. El segundo homomorfismo, que denotaremos por $\pi$ es dado por $\pi(x)=\left(\frac{x}{1}, \ldots, \frac{x}{1}\right) \in \bigoplus_{i=1}^{k} M_{a_{i}, \varphi}$ para todo $x \in M$. Debemos probar que $\operatorname{ker}(\pi)=\operatorname{im}(\iota) . \operatorname{En}$ efecto, si $x \in \Gamma_{\mathfrak{a}, \varphi}(M)$, entonces existe un número natural $n \geq 1$ y un ideal $\mathfrak{b} \in \varphi$ de manera que $\operatorname{Ann}(x)+\mathfrak{b} \supseteq \mathfrak{a}^{n}$. Luego, para cada $i=1, \ldots, k$ podemos escribir $a_{i}^{n}=r_{i}+b_{i}$ con $r_{i} \in \operatorname{Ann}(x)$ y $b_{i} \in \mathfrak{b}$. Por lo tanto, $a_{i}^{n} x=b_{i} x$, o equivalentemente, $\left(a_{i}^{n}+\left(-b_{i}\right)\right) x=0$. Desde que $a_{i}^{n}+\left(-b_{i}\right) \in S_{a_{i}, \varphi}$, la última relación muestra que $\pi(x)=0$. Recíprocamente, supongamos que $x \in \operatorname{ker}(\pi)$. Entonces para cada $i=1, \ldots, k$ existe un entero no negativo $n_{i}$, un ideal $\mathfrak{b}_{i} \in \varphi$ y un elemento $b_{i} \in \mathfrak{b}_{i}$ tal que $\left(a_{i}^{n_{i}}+b_{i}\right) x=0$. De esa forma, $r_{i}:=a_{i}^{n_{i}}+b_{i} \in \operatorname{Ann}(x)$. Por lo tanto, si $n:=n_{1}+\cdots+n_{k}$ y $\mathfrak{b}=\mathfrak{b}_{1}+\cdots+\mathfrak{b}_{k} \in \varphi$, las relaciones anteriores implican que $\mathfrak{a}^{n} \subseteq \operatorname{Ann}(x)+\mathfrak{b}$. En consecuencia, $x \in \Gamma_{\mathfrak{a}, \varphi}$.

\subsection{Los funtores de cohomología $\mathrm{H}_{\mathfrak{a}, \varphi}^{i}(-)$}

Definición 3.2.1. Para cada entero no negativo $i \geq 0$, el $i$-ésimo funtor de cohomología local con respecto al par $(\mathfrak{a}, \varphi)$, denotado por $\mathrm{H}_{\mathfrak{a}, \varphi}^{i}(-)$, es definido como $i$-ésimo funtor derivado a la derecha del $(\mathfrak{a}, \varphi)$ - funtor de torsión $\Gamma_{\mathfrak{a}, \varphi}(-)$, i.e.,

$$
\mathrm{H}_{\mathfrak{a}, \varphi}^{i}(-):=\mathcal{R}^{i} \Gamma_{\mathfrak{a}, \varphi}(-) .
$$

Por lo visto en la sección, las siguientes propiedades caracterizan a los funtores $\left\{H_{\mathfrak{a}, \varphi}^{i}(-)\right\}_{i \geq 0}$.

i) Los funtores $\mathrm{H}_{\mathfrak{a}, \varphi}^{0}(-)$ y $\Gamma_{\mathfrak{a}, \varphi}(-)$ son isomorfos naturalmente.

ii) $\mathrm{H}_{\mathfrak{a}, \varphi}^{i}(E)=0$ para todo módulo inyectivo $E$ y para todo entero positivo $i>0$.

iii) Toda sucesión exacta corta de módulos

$$
0 \longrightarrow L \longrightarrow M \longrightarrow N \longrightarrow 0
$$

induce una sucesión exacta larga natural

$$
0 \longrightarrow \Gamma_{\mathfrak{a}, \varphi}(L) \longrightarrow \Gamma_{\mathfrak{a}, \varphi}(M) \longrightarrow \Gamma_{\mathfrak{a}, \varphi}(N) \stackrel{\partial}{\longrightarrow} \mathrm{H}_{\mathfrak{a}, \varphi}^{1}(L) \longrightarrow \mathrm{H}_{\mathfrak{a}, \varphi}^{1}(M) \longrightarrow \cdots
$$

Proposición 3.2.1. Sea $M$ un A-módulo. Si $a \in \mathfrak{a}$, entonces

$$
\mathrm{H}_{\mathfrak{a}, \varphi}^{i}\left(M_{a, \varphi}\right)=0 \text { para todo } i \geq 0 .
$$


Prueba. Sea $I^{\bullet}$ una resolución inyectiva de $M$, entonces $\left(I^{\bullet}\right)_{a, \varphi}$ es una resolución inyectiva de $M_{a, \varphi}$. Luego, $\mathrm{H}_{\mathfrak{a}, \varphi}^{i}\left(M_{a, \varphi}\right)=\mathrm{H}^{i}\left(\Gamma_{\mathfrak{a}, \varphi}\left(\left(I^{\bullet}\right)_{a, \varphi}\right)\right)$. Ahora escriba cada $I^{i}$ como suma directa de módulos inyectivos irreducibles, $I^{i}=\bigoplus_{\mathfrak{p} \in \operatorname{Spec}(A)} E_{A}(A / \mathfrak{p})^{\mu_{i}(\mathfrak{p}, M)}$. Entonces

$$
\left(I^{i}\right)_{a, \varphi} \cong \bigoplus_{\mathfrak{p} \in \operatorname{Spec}(A)} E_{A}(A / \mathfrak{p})_{a, \varphi}^{\mu_{i}(\mathfrak{p}, M)} \cong \bigoplus_{\mathfrak{p} \in \operatorname{Spec}(A)} E_{A_{a, \varphi}}\left(A_{a, \varphi} / \mathfrak{p} A_{a, \varphi}\right)^{\mu_{i}(\mathfrak{p}, M)}
$$

Por lo tanto, gracias a la parte ii) del Lema 3.1 .2 , obtenemos

$$
\Gamma_{a, \varphi}\left(I^{i}\right)_{a, \varphi} \cong \bigoplus_{\mathfrak{p} \in \mathcal{G}(\mathfrak{a}, \varphi)} \Gamma_{a, \varphi}\left(E_{A_{a, \varphi}}\left(A_{a, \varphi} / \mathfrak{p} A_{a, \varphi}\right)^{\mu_{i}(\mathfrak{p}, M)}\right)=0
$$

En consecuencia, $\mathrm{H}_{\mathfrak{a}, \varphi}^{i}\left(M_{a, \varphi}\right)=0$ para todo entero no negativo $i$.

Necesitaremos del siguiente resultado sobre localizaciones.

Lema 3.2.1. Sean $S$ y $T$ dos subconjuntos multiplicativos de A, entonces existe un isomorfismo de A-álgebras

$$
A_{S} \otimes_{A} A_{T} \cong\left(A_{S}\right)_{\bar{T}}
$$

donde $\bar{T}:=\iota(T)$ es la imagen de $T$ vía el homomorfismo de localización $\iota: A \longrightarrow A_{S}$.

Prueba. Consideremos el homomorfismo de $A$-álgebras $\sigma: A \longrightarrow A_{S} \otimes_{A} A_{T}$ definido por $\sigma(a)=$ $\iota(a) \otimes 1$. Notemos que si $a \in S$, entonces $\sigma(a)$ es una unidad de $A_{S} \otimes_{A} A_{T}$. Luego, por la propiedad universal de $A_{S}$, existe un único homomorfismo de $A$-álgebras

$\alpha: A_{S} \longrightarrow A_{S} \otimes_{A} A_{T}$ tal que el siguiente diagrama es conmutativo



Observemos que para cualquier elemento $t \in T, \alpha(\iota(t))=\sigma(t)=t \sigma(1)=1 \otimes \frac{t}{1}$ es una unidad de $A_{S} \otimes_{A} A_{T}$. Denotemos por $j: A_{S} \longrightarrow\left(A_{S}\right)_{\bar{T}}$ el homomorfismo de localización. La propiedad universal de $\left(A_{S}\right)_{\bar{T}}$ garantiza la existencia de un único homomorfismo de $A$-álgebras $\beta:\left(A_{S}\right)_{\bar{T}} \longrightarrow A_{S} \otimes_{A} A_{T}$ tal que el diagrama que sigue es conmutativo.

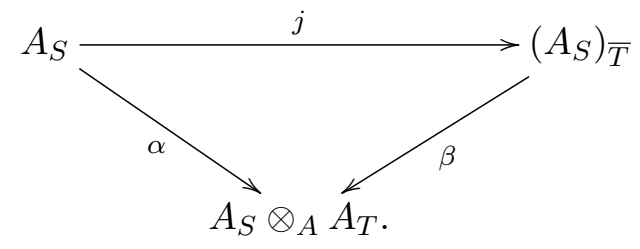

Sea $k: A \longrightarrow A_{T}$ el homomorfismo de localización. Desde que la composición $j \circ \iota: A \longrightarrow\left(A_{S}\right)_{\bar{T}}$ lleva elementos de $T$ en unidades de $\left(A_{S}\right)_{\bar{T}}$, la propiedad universal de $A_{T}$ implica que existe un único homomorfismo de $A$-álgebras $\varphi: A_{T} \longrightarrow\left(A_{S}\right)_{\bar{T}}$ tal que el siguiente diagrama es conmutativo.

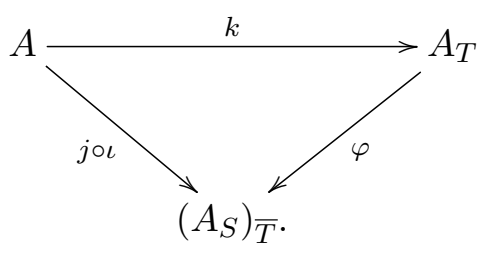


Consideremos ahora la aplicación $A$-bilineal $\gamma: A_{S} \times A_{T} \longrightarrow\left(A_{S}\right)_{\bar{T}}$ definida por $\gamma(x, y)=$ $j(x) \varphi(y)$. Entonces, por la propiedad universal del producto tensorial $A_{S} \otimes_{A} A_{T}$, existe un único homomorfismo $A$-lineal $\theta: A_{S} \otimes_{A} A_{T} \longrightarrow\left(A_{S}\right)_{\bar{T}}$ de manera tal que el siguente diagrama conmuta.

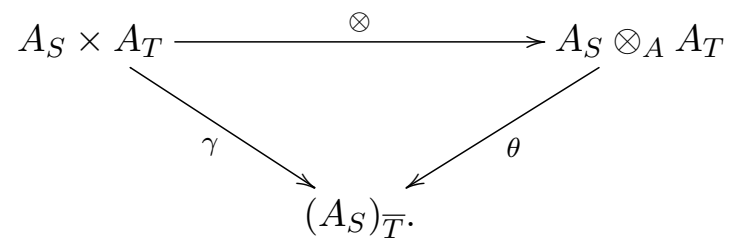

Ahora no es difícil verificar que $\beta \circ \theta=$ id y $\theta \circ \beta=$ id.

Definición 3.2.2. Para un elemento $a$ de $A$ y un conjunto no vacío de ideales $\varphi$ de $A$, definimos el complejo $\mathcal{C}_{a, \varphi}^{\bullet}$ por

$$
\mathcal{C}_{a, \varphi}^{\bullet}: \quad 0 \longrightarrow \underbrace{A}_{0} \stackrel{\iota}{\longrightarrow} \underbrace{A_{a, \varphi}}_{1} \longrightarrow 0,
$$

donde $A$ está colocado en la posición $0, A_{a, \varphi}$ en la posición 1, y $\iota$ es la aplicación de localización.

Observemos que para un módulo $M$ sobre $A$,

$$
\begin{aligned}
\mathrm{H}^{0}\left(\mathcal{C}_{a, \varphi}^{\bullet} \otimes_{A} M\right) & =\operatorname{ker}(\iota \otimes M) \\
& =\left\{x \in M: r x=0 \text { para algún } r \in S_{a, \varphi}\right\} \\
& =\left\{x \in M:\left(a^{n}+b\right) x=0 \text { para algunos } n \geq 1, \mathfrak{b} \in\langle\varphi\rangle \text { con } b \in \mathfrak{b}\right\} \\
& =\left\{x \in M: a^{n} \in \operatorname{Ann}(x)+\mathfrak{b} \text { para algunos } n \geq 1, \mathfrak{b} \in\langle\varphi\rangle\right\} \\
& =\Gamma_{\mathfrak{a}, \varphi}(M),
\end{aligned}
$$

donde $\mathfrak{a}$ es el ideal principal generado por $a$.

Ahora podemos definir el complejo generalizado de Čech de la siguiente manera:

Definición 3.2.3. Para una sucesión finita $\bar{a}=a_{1}, \ldots, a_{n}$ de elementos de $A$ y un conjunto no vacío $\varphi$ de ideales de $A$, el complejo de $A$-módulos $\mathcal{C}_{\bar{a}, \varphi}$ es definido como el complejo producto tensorial de los complejos $\mathcal{C}_{a_{i}, \varphi}^{\bullet}$, i.e.,

$$
\mathcal{C}_{\bar{a}, \varphi}^{\bullet}:=\bigotimes_{i=1}^{n} \mathcal{C}_{a_{i}, \varphi}^{\bullet}
$$

Por ejemplo, como consecuencia del Lema 3.2.1 para $n=2$ tenemos el siguiente complejo

$$
\mathcal{C}^{\bullet}{ }_{\left(a_{1}, a_{2}\right), \varphi}: \quad 0 \longrightarrow A \longrightarrow A_{a_{1}, \varphi} \oplus A_{a_{2}, \varphi} \longrightarrow\left(A_{a_{1}, \varphi}\right)_{a_{2}, \varphi} \longrightarrow 0
$$

De manera más general, $\mathcal{C}^{\bullet} \bar{a}, \varphi$ toma la siguiente forma:

$$
0 \longrightarrow A \longrightarrow \bigoplus_{i=1}^{n} A_{a_{i}, \varphi} \longrightarrow \bigoplus_{i<j}\left(A_{a_{i}, \varphi}\right)_{a_{j}, \varphi} \longrightarrow \cdots \longrightarrow\left(\cdots A_{a_{1}, \varphi} \cdots\right)_{a_{n}, \varphi} \longrightarrow 0
$$

En el próximo lema usaremos la siguiente notación: Dada una sucesión $\bar{a}=a_{1}, \ldots, a_{n}$ de elementos de $A$ y un elemento $b \in A$, denotaremos por $\bar{a} \cup b$ la sucesión $a_{1}, \ldots, a_{n}, b$.

Lema 3.2.2. Sean $\varphi$ un conjunto no vacío de ideales de $A$ y $M$ un A-módulo. Existe una sucesión exacta corta de complejos de A-módulos.

$$
0 \longrightarrow \mathcal{C}_{\bar{a}, \varphi}[-1] \otimes M_{b, \varphi} \longrightarrow \mathcal{C}_{\bar{a} \cup b, \varphi} \otimes M \longrightarrow \mathcal{C}_{\bar{a}, \varphi} \otimes M \longrightarrow 0
$$


Prueba. El siguiente diagrama

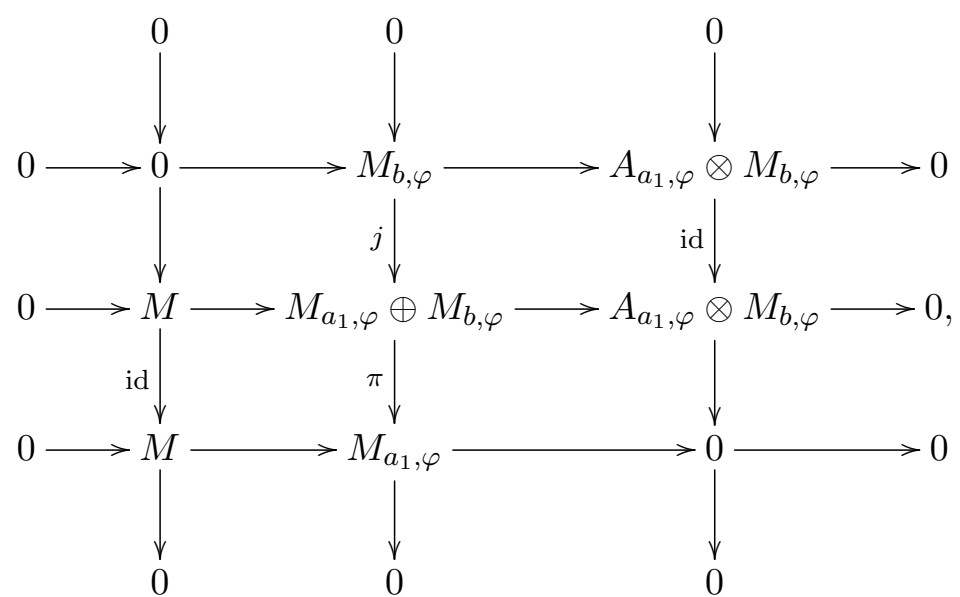

donde $j(x)=(0, x)$ y $\pi(x, y)=x$, es un diagrama conmutativo cuyas columnas son exactas. Esto demuestra el lema cuando $n=1$. Ahora el caso general puede ser demostrado fácilmente por inducción sobre $n$.

El siguente resultado generaliza [9, Theorem 2.4], y permite calcular los módulos de cohomología local soportados en el sistema de ideales $\mathcal{G}(\mathfrak{a}, \varphi)$ en términos de los módulos de cohomología del complejo generalizado de Čech.

Teorema 3.2.1. Sea $\bar{a}=a_{1}, \ldots, a_{n}$ una sucesión finita de elementos de $A$ y $\varphi$ un conjunto no vacio de ideales de $A$. Para todo $i \geq 0$ existe un isomorfismo natural de funtores

$$
\mathrm{H}_{\mathfrak{a}, \varphi}^{i}(-) \cong \mathrm{H}^{i}\left(\mathcal{C}_{\bar{a}, \varphi} \otimes_{A}-\right),
$$

donde $\mathfrak{a}=\left\langle a_{1}, \ldots, a_{n}\right\rangle$ es el ideal generado por la sucesión $\bar{a}$.

Prueba. La parte iii) del Lema 3.1.2 garantiza la existencia de un isomorfismo natural de funtores

$$
\mathrm{H}^{0}\left(\mathcal{C}_{\bar{a}, \varphi} \otimes_{A}-\right) \cong \Gamma_{\mathfrak{a}, \varphi}(-) .
$$

Como la sucesión $\left\{\mathrm{H}^{i}\left(\mathcal{C}_{\bar{a}, \varphi} \otimes_{A}-\right)\right\}_{i \geq 0}$ es un $\delta$-funtor cohomológico, para demostrar el Teorema es suficiente mostrar que $\mathrm{H}^{i}\left(\mathcal{C}_{\bar{a}, \varphi} \otimes_{A} I\right)=0$ para todo $A$-módulo inyectivo $I$ y para todo $i \geq 1$. A partir de la descomposición $I \cong \bigoplus_{\mathfrak{p} \in \operatorname{Spec}(A)} E_{A}(A / \mathfrak{p})$ y del hecho que cada funtor $\mathrm{H}^{i}\left(\mathcal{C}^{\bullet} \bar{a}, \varphi \otimes_{A}-\right)$ preserva sumas directas, podemos suponer sin pérdida de generalidad que $I=E_{A}(A / \mathfrak{p})$ para algún ideal primo p. Procederemos por inducción sobre $n$, el número de elementos de la sucesión $\bar{a}$.

Si $n=1$, entonces el complejo de $A$-módulos $\mathcal{C}_{a_{1}, \varphi}^{\bullet} \otimes_{A} E_{A}(A / \mathfrak{p})$ es dado por

$$
0 \longrightarrow E_{A}(A / \mathfrak{p}) \stackrel{\iota}{\longrightarrow} E_{A}(A / \mathfrak{p})_{a_{1}, \varphi} \longrightarrow 0
$$

Tenemos las siguientes posibilidades: si $\mathfrak{p} \in \mathcal{G}\left(\left\langle a_{1}\right\rangle, \varphi\right)$, entonces por la parte ii) del Lema 3.1.2, $\mathfrak{p} \cap S_{a, \varphi} \neq \emptyset$. Por lo tanto, $E_{A}(A / \mathfrak{p})_{a_{1}, \varphi}=0$ (ver [즈, Proposition 10.1.14 (i)]. Por otro lado, si $\mathfrak{p} \notin \mathcal{G}\left(\left\langle a_{1}\right\rangle, \varphi\right)$, entonces $\mathfrak{p} \cap S_{a_{1}, \varphi}=\emptyset$. Caso contrario, existe un elemento $c \in \mathfrak{p} \cap S_{a_{1}, \varphi}$. Por lo tanto podemos escribir $c=a_{1}^{n}+b$, para algún $\mathfrak{b} \in\langle\varphi\rangle$ con $b \in \mathfrak{b}$. Así, $a_{1}^{n}=(-b)+c \in \mathfrak{b}+\mathfrak{p}$. Luego $\mathfrak{p} \in \mathcal{G}\left(\left\langle a_{1}\right\rangle, \varphi\right)$, lo que contradice nuestra suposición. Como consecuencia de [3, Proposition 10.1 .14 (i)], $E_{A}(A / \mathfrak{p}) \cong E_{A}(A / \mathfrak{p})_{a_{1}, \varphi}$ como $A$-módulos. En cualquiera de los casos, obtenemos que $\mathrm{H}^{1}\left(\mathcal{C}_{a_{1}, \varphi}^{\bullet} \otimes_{A} E_{A}(A / \mathfrak{p})\right)=0$.

Ahora supongamos que $n \geq 1$ y que el resultado vale para valores menores o iguales que $n$. Sea 
$a_{1}, \ldots, a_{n}, a_{n+1}$ una sucesión finita de elementos de $A$. Denote por $\bar{a}$ la sucesión $a_{1}, \ldots, a_{n}$. Por el Lema 3.2 .2 existe una sucesión exacta corta de complejos de $A$-módulos

$$
0 \longrightarrow \mathcal{C}_{\bar{a}, \varphi}[-1] \otimes E_{A}(A / \mathfrak{p})_{a_{n+1}, \varphi} \longrightarrow \mathcal{C}^{\bullet}{ }_{\bar{a} \cup a_{n+1}, \varphi} \otimes E_{A}(A / \mathfrak{p}) \longrightarrow \mathcal{C}_{\bar{a}, \varphi} \otimes E_{A}(A / \mathfrak{p}) \longrightarrow 0 .
$$

Tal sucesión induce una sucesión exacta larga de $A$-módulos de cohomología

$$
\begin{aligned}
& 0 \rightarrow \mathrm{H}^{0}\left(\mathcal{C}_{\bar{a}, \varphi}[-1] \otimes E_{A}(A / \mathfrak{p})_{a_{n+1}, \varphi}\right) \rightarrow \mathrm{H}^{0}\left(\mathcal{C}_{\bar{a} \cup a_{n+1}, \varphi} \otimes E_{A}(A / \mathfrak{p})\right) \rightarrow \mathrm{H}^{0}\left(\mathcal{C}_{\bar{a}, \varphi} \otimes E_{A}(A / \mathfrak{p})\right) \\
& \stackrel{\delta^{0}}{\rightarrow} \mathrm{H}^{1}\left(\mathcal{C}_{\bar{a}, \varphi}[-1] \otimes E_{A}(A / \mathfrak{p})_{a_{n+1}, \varphi}\right) \rightarrow \mathrm{H}^{1}\left(\mathcal{C}^{\bullet}{ }_{\bar{a} \cup a_{n+1}, \varphi} \otimes E_{A}(A / \mathfrak{p})\right) \rightarrow \underbrace{\mathrm{H}^{1}\left(\mathcal{C}_{\bar{a}, \varphi} \otimes E_{A}(A / \mathfrak{p})\right)}_{0} \\
& \stackrel{\delta^{1}}{\rightarrow} \underbrace{\mathrm{H}^{2}\left(\mathcal{C}_{\bar{a}, \varphi}^{\bullet}[-1] \otimes E_{A}(A / \mathfrak{p})_{a_{n+1}, \varphi}\right)}_{0} \rightarrow \mathrm{H}^{2}\left(\mathcal{C}_{\bar{a} \cup a_{n+1}, \varphi} \otimes E_{A}(A / \mathfrak{p})\right) \rightarrow \underbrace{\mathrm{H}^{2}\left(\mathcal{C}_{\bar{a}, \varphi}^{\bullet} \otimes E_{A}(A / \mathfrak{p})\right)}_{0} \rightarrow \cdots
\end{aligned}
$$

Desde que $E_{A}(A / \mathfrak{p})_{a_{n+1}, \varphi}$ es un módulo inyectivo, deducimos a partir de la hipótesis inductiva que

$$
\mathrm{H}^{i}\left(\mathcal{C}_{\bar{a}, \varphi}^{\bullet}[-1] \otimes E_{A}(A / \mathfrak{p})_{a_{n+1}, \varphi}\right)=0 \text { para todo } i \geq 2
$$

$\mathrm{y}$

$$
\mathrm{H}^{i}\left(\mathcal{C}_{\bar{a}, \varphi} \otimes E_{A}(A / \mathfrak{p})\right)=0 \text { para todo } i \geq 1
$$

Esto implica que

$$
\mathrm{H}^{i}\left(\mathcal{C}^{\bullet}{ }_{\bar{a} \cup a_{n+1}, \varphi} \otimes E_{A}(A / \mathfrak{p})\right)=0 \text { para todo } i \geq 2 .
$$

Finalmente, como

$$
\mathrm{H}^{0}\left(\mathcal{C}_{\bar{a}, \varphi} \otimes E_{A}(A / \mathfrak{p})\right) \cong \Gamma_{\mathfrak{a}, \varphi}\left(E_{A}(A / \mathfrak{p})\right)
$$

$\mathrm{y}$

$$
\mathrm{H}^{1}\left(\mathcal{C}_{\bar{a}, \varphi}^{\bullet}[-1] \otimes E_{A}(A / \mathfrak{p})_{a_{n+1}, \varphi}\right)=\mathrm{H}^{0}\left(\mathcal{C}_{\bar{a}, \varphi}^{\bullet} \otimes E_{A}(A / \mathfrak{p})_{a_{n+1}}\right) \cong \Gamma_{\mathfrak{a}, \varphi}\left(E_{A}(A / \mathfrak{p})_{a_{n+1}}\right),
$$

vemos que $\delta^{0}=\Gamma_{\mathfrak{a}, \varphi}(f)$, donde $f: E_{A}(A / \mathfrak{p}) \longrightarrow E_{A}(A / \mathfrak{p})_{a_{n+1}}$ es el mapa de localización. Consideremos ahora el conjunto multiplicativo $T:=\left\{1, a_{n+1}, a_{n+1}^{2}, \ldots\right\}$. Tenemos dos posibilidades: Si $\mathfrak{p} \cap T \neq \emptyset$, entonces por [3, Proposition 10.1.14 (i)], $E_{A}(A / \mathfrak{p})_{a_{n+1}}=0$. Por otro lado, si $\mathfrak{p} \cap T=\emptyset$, entonces por [3, Proposition 10.1.14 (i)], $E_{A}(A / \mathfrak{p})_{a_{n+1}, \varphi} \cong E_{A}(A / \mathfrak{p})$ como $A$-módulos. En cualquier caso, la sucesión exacta larga permite concluir que

$$
\mathrm{H}^{1}\left(\mathcal{C}^{\bullet}{\bar{a} \cup a_{n+1}, \varphi} \otimes E_{A}(A / \mathfrak{p})\right)=0 .
$$

Esto completa el proceso inductivo, y por lo tanto, la demostración del Teorema.

Observación 3.2.1. Para completar el paso inductivo, también podemos proceder de la siguiente manera: Desde que $\mathcal{C}^{\bullet}{ }_{\bar{a} \cup a_{n+1}}=\mathcal{C}^{\bullet}{ }_{a_{n+1}} \otimes \mathcal{C}^{\bullet} \bar{a}$, existe una sucesión espectral

$$
\mathrm{E}_{2}^{p, q}=\mathrm{H}^{p}\left(\mathcal{C}^{\bullet}{ }_{a_{n+1}} \otimes \mathrm{H}^{q}\left(\mathcal{C}^{\bullet}{ }_{\bar{a}} \otimes E_{A}(A / \mathfrak{p})\right)\right) \Rightarrow \mathrm{H}^{p+q}\left(\mathcal{C}_{\bar{a} \cup a_{n+1}} \otimes E_{A}(A / \mathfrak{p})\right) .
$$

En vista que $\mathrm{H}^{q}\left(\mathcal{C}_{\bar{a}} \otimes E_{A}(A / \mathfrak{p})\right)=0$ para todo $q \geq 1$ por causa de la hipótesis inductiva, la sucesión espectral degenera. Por lo tanto tenemos los siguientes isomorfismos

$$
\begin{aligned}
\mathrm{H}^{i}\left(\mathcal{C}_{\bar{a} \cup a_{n+1}} \otimes E_{A}(A / \mathfrak{p})\right) & \cong \mathrm{H}^{i}\left(\mathcal{C}_{a_{n+1}} \otimes \mathrm{H}^{0}\left(\mathcal{C}_{\bar{a}}^{\bullet} \otimes E_{A}(A / \mathfrak{p})\right)\right) \\
& \cong \mathrm{H}^{i}\left(\mathcal{C}_{a_{n+1}}^{\bullet} \otimes \Gamma_{\mathfrak{a}, \varphi}\left(E_{A}(A / \mathfrak{p})\right)\right) \\
& =\mathrm{H}^{i}\left(0 \longrightarrow \Gamma _ { \mathfrak { a } , \varphi } \left(E_{A}(A / \mathfrak{p}) \longrightarrow \Gamma_{\mathfrak{a}, \varphi}\left(E_{A}(A / \mathfrak{p})_{a_{n+1}} \longrightarrow 0\right) .\right.\right.
\end{aligned}
$$

Esto implica que

$$
\mathrm{H}^{i}\left(\mathcal{C}_{\bar{a} \cup a_{n+1}, \varphi} \otimes E_{A}(A / \mathfrak{p})\right)=0 \text { para todo } i \geq 2 \text {. }
$$


Finalmente, si $\mathfrak{p} \notin \mathcal{G}(\mathfrak{a}, \varphi)$ entonces $\Gamma_{\mathfrak{a}, \varphi}\left(E_{A}(A / \mathfrak{p})=0\right.$. Por otro lado, si $\mathfrak{p} \in \mathcal{G}(\mathfrak{a}, \varphi)$, entonces $\Gamma_{\mathfrak{a}, \varphi}\left(E_{A}(A / \mathfrak{p})\right)=E_{A}(A / \mathfrak{p})$. Luego podemos usar el mismo argumento que en la parte final de la demostración del Teorema para concluir que

$$
\mathrm{H}^{1}\left(\mathcal{C}^{\bullet}{\bar{a} \cup a_{n+1}, \varphi} \otimes E_{A}(A / \mathfrak{p})\right)=0 .
$$

\subsection{Homología local}

Dada una sucesión finita $\bar{a}=a_{1}, \ldots, a_{n}$ de elementos de $A$, un conjunto no vacío $\varphi$ de ideales de $A$ y un $A$-módulo $M$, definimos el co-complejo de Cech generalizado de $M$ como el complejo de $A$-módulos $\operatorname{Hom}\left(\mathcal{C}_{\bar{a}, \varphi}^{\bullet}, M\right)$.

Definición 3.3.1. Para cada $i \geq 0$ definimos el $i$-ésimo funtor de homología local con respecto al $\operatorname{par}(\mathfrak{a}, \varphi)$ por

$$
\mathrm{H}_{i}^{\mathfrak{a}, \varphi}(-):=\mathrm{H}_{i}\left(\operatorname{Hom}\left(\mathcal{C}_{\bar{a}, \varphi}^{\bullet},-\right)\right)
$$

De esa forma, para cada $A$-módulo $M$,

$$
\mathrm{H}_{i}^{\mathfrak{a}, \varphi}(M):=\mathrm{H}_{i}\left(\operatorname{Hom}\left(\mathcal{C}_{\bar{a}, \varphi}^{\bullet}, M\right)\right) .
$$

Por ejemplo, cuando $n=1$ y $n=2$, la homología local con respecto al par $(\mathfrak{a}, \varphi)$ puede ser calculada como la homología de los siguientes complejos, respectivamente.

$$
0 \longrightarrow \operatorname{Hom}_{A}\left(A_{a_{1}, \varphi}, M\right) \longrightarrow M \longrightarrow 0
$$

$\mathrm{y}$

$$
\left.0 \longrightarrow \operatorname{Hom}_{A}\left(A_{a_{1}, \varphi}\right)_{a_{2}, \varphi}, M\right) \longrightarrow \operatorname{Hom}_{A}\left(A_{a_{1}, \varphi}, M\right) \oplus \operatorname{Hom}_{A}\left(A_{a_{2}, \varphi}, M\right) \longrightarrow M \longrightarrow 0
$$

Lema 3.3.1. Sean $E$ y $N$ dos módulos sobre $A$ tales que $E$ es inyectivo y $N$ es finitamente generado. Para cada $i \geq 0$ existe un isomorfismo natural

$$
\operatorname{Tor}_{i}^{A}\left(N, D_{E}(-)\right) \cong D_{E}\left(\operatorname{Ext}_{\mathrm{A}}^{\mathrm{i}}(\mathrm{N},-)\right) \text {. }
$$

Prueba. Sea $\mathcal{P}_{\bullet}$ una resolución libre de $N$ tal que cada término de $\mathcal{P}_{\bullet}$ es de rango finito. Luego,

$$
\begin{aligned}
\operatorname{Tor}_{i}^{A}\left(N, D_{E}(-)\right) & =\operatorname{Tor}_{i}^{A}\left(N, \operatorname{Hom}_{A}(-, E)\right) \\
& =\mathrm{H}_{i}\left(\mathcal{P} \bullet \otimes \operatorname{Hom}_{A}(-, E)\right) \\
& \cong \mathrm{H}_{i}\left(\operatorname{Hom}_{A}\left(\operatorname{Hom}_{A}(\mathcal{P} \bullet,-), E\right)\right) \\
& \cong \operatorname{Hom}_{A}\left(H^{i}(\operatorname{Hom}(\mathcal{P} \bullet,-)), E\right) \\
& =\operatorname{Hom}_{A}\left(\operatorname{Ext}_{\mathrm{A}}^{\mathrm{i}}(N,-), E\right) \\
& =D_{E}\left(\operatorname{Ext}_{A}^{i}(N,-)\right) .
\end{aligned}
$$

Antes de probar nuestro próximo Teorema, recordemos que un $A$ - módulo $E$ es un cogenerador inyectivo de $A$ si el funtor

$$
\operatorname{Hom}_{A}(-, E):\left(\operatorname{Mod}_{A}\right)^{\mathrm{op}} \longrightarrow \operatorname{Mod}_{A}
$$

es exacto y fiel. El principal ejemplo de un cogenerador inyectivo de $A$ es dado por

$$
E_{A}\left(\bigoplus_{\mathfrak{m} \in \operatorname{Max}(A)} A / \mathfrak{m}\right),
$$


donde, como es usual, $E_{A}(M)$ denota la cápsula inyectiva de un $A$-módulo $M$. Cuando $A$ es un anillo Noetheriano vale la igualdad

$$
E_{A}\left(\bigoplus_{\mathfrak{m} \in \operatorname{Max}(A)} A / \mathfrak{m}\right)=\bigoplus_{\mathfrak{m} \in \operatorname{Max}(A)} E_{A}(A / \mathfrak{m})
$$

A continuación, fijemos un cogenerador inyectivo $E$ de $A$ y denotemos por $D_{E}(-)$ el funtor (contravariante) $\operatorname{Hom}_{A}(-, E)$. Notemos que existe una transformación natural

$$
\theta: \operatorname{id}(-) \longrightarrow D_{E} D_{E}(-)
$$

que es inyectiva para cada $A$-módulo $M$. A saber,

$$
\begin{aligned}
\theta_{M}: M & \longrightarrow \operatorname{Hom}_{A}\left(\operatorname{Hom}_{A}(M, E), E\right) \\
x & \longmapsto \theta_{M}(x): \varphi \longmapsto \varphi(x) .
\end{aligned}
$$

La inyectividad de $\theta_{M}$ es consecuencia del siguiente diagrama conmutativo

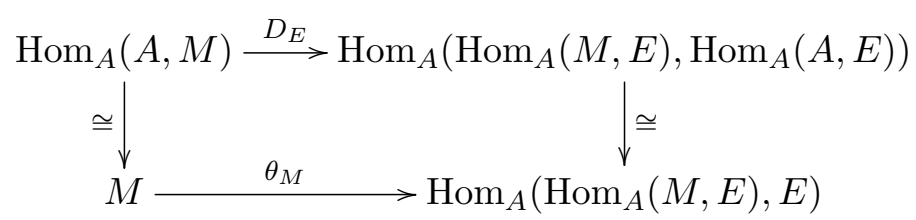

y del hecho que la primera fila es inyectiva, a raiz que $D_{E}(-)$ es un funtor fiel.

Definición 3.3.2. Decimos que un $A$-módulo $M$ es $E$-Matlis reflexivo si

$$
\theta_{M}: M \longrightarrow D_{E} D_{E}(M)
$$

es una biyección. Cuando el anillo $A$ es Noetheriano y $E=E_{A}\left(\bigoplus_{\mathfrak{m} \in \operatorname{Max}(A)} A / \mathfrak{m}\right)$, simplemente decimos que $M$ es Matlis reflexivo.

Es importante observar que los módulos Matlis reflexivos fueron introducidos y caracterizados por R. G. Belshoff, E. E. Enochs y J. R. García Rozas en [2].

Proposición 3.3.1. Un A-módulo $M$ es E-Matlis reflexivo, si y solo si, $D_{E}(M)$ es E-Matlis reflexivo.

Prueba. Supongamos que $M$ es $E$-Matlis reflexivo. Consideremos el homomorfismo $A$-lineal $\theta_{D_{E}(M)}: D_{E}(M) \longrightarrow D_{E} D_{E} D_{E}(M)$. Tome un elemento $\varphi \in D_{E} D_{E} D_{E}(M)$, entonces $\varphi \circ \theta_{M} \in$ $D_{E}(M)$. Afirmamos que $\theta_{D_{E}(M)}\left(\varphi \circ \theta_{M}\right)=\varphi$. En efecto, sea $\beta \in D_{E} D_{E} M$, entonces existe $x \in M$ tal que $\beta=\theta_{M}(x)$. Luego,

$$
\theta_{D_{E}(M)}\left(\varphi \circ \theta_{M}\right)(\beta)=\beta\left(\varphi \circ \theta_{M}\right)=\theta_{M}(x)\left(\varphi \circ \theta_{M}\right)=\varphi\left(\theta_{M}(x)\right)=\varphi(\beta) .
$$

Por lo tanto, $\theta_{D_{E}(M)}$ es sobreyectivo, y en consecuencia, $D_{E}(M)$ es $E$-Matlis reflexivo.

Recíprocamente, supongamos que $D_{E}(M)$ es $E$-Matlis reflexivo. Observemos que $D_{E}\left(\theta_{M}\right) \circ$ $\theta_{D_{E}(M)}=\operatorname{id}_{D_{E}(M)}$. Desde que $\theta_{D_{E}(M)}$ es un isomorfismo tenemos que $D_{E}\left(\theta_{M}\right)$ también es un isomorfismo. Como $D_{E}(-)$ es un funtor exacto y fiel, concluímos que $\theta_{M}$ es un isomorfismo. Por lo tanto, $M$ es $E$-Matlis reflexivo.

Teorema 3.3.1. Sean $E$ un cogenerador inyectivo de $A$ y $M$ un A-módulo E-Matlis reflexivo, entonces para cada $i \geq 0$, existe un isomorfismo natural

$$
\mathrm{H}_{i}^{\mathfrak{a}, \varphi}(M) \cong \lim _{\mathfrak{b} \in \mathscr{\mathcal { G } ( \mathfrak { a } , \varphi )}} \operatorname{Tor}_{i}^{A}(A / \mathfrak{b}, M)
$$


Prueba. Por la definición de módulo $E$-Matlis reflexivo, $D_{E} D_{E}(M) \cong M$. Aplicando el Corolario 2.3.1 y el Lema 3.3.1, obtenemos los siguientes isomorfismos naturales

$$
\begin{aligned}
& \mathrm{H}_{i}^{\mathfrak{a}, \varphi}(M)=\mathrm{H}^{i}\left(\operatorname{Hom}_{A}\left(\mathcal{C}_{\bar{a}, \varphi}, M\right)\right. \\
& \cong \mathrm{H}_{i}\left(\operatorname{Hom}_{A}\left(\mathcal{C}_{\bar{a}, \varphi}, D_{E} D_{E} M\right)\right. \\
& \cong \mathrm{H}_{i}\left(\operatorname{Hom}_{A}\left(\mathcal{C}_{\bar{a}, \varphi} \otimes_{A} D_{E} M, E\right)\right) \\
& \cong D_{E}\left(\mathrm{H}^{i}\left(\mathcal{C}_{\bar{a}, \varphi} \otimes_{A} D_{E} M\right)\right) \\
& \cong D_{E}\left(\mathrm{H}_{\mathfrak{a}, \varphi}^{i}\left(D_{E} M\right)\right)
\end{aligned}
$$

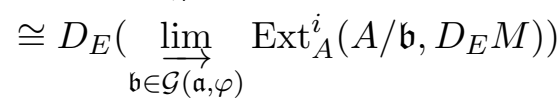

$$
\begin{aligned}
& \cong \lim _{\mathfrak{b} \in \overleftarrow{\mathcal{G}(\mathfrak{a}, \varphi)}} D_{E}\left(\operatorname{Ext}_{A}^{i}\left(A / \mathfrak{b}, D_{E} M\right)\right) \\
& \cong \lim _{\mathfrak{b} \in \overleftarrow{\mathcal{G}(\mathfrak{a}, \varphi)}} \operatorname{Tor}_{i}^{A}\left(A / \mathfrak{b}, D_{E} D_{E} M\right) \\
& \cong \lim _{\mathfrak{b} \in \overleftarrow{\mathcal{G}(\mathfrak{a}, \varphi)}} \operatorname{Tor}_{i}^{A}(A / \mathfrak{b}, M)
\end{aligned}
$$

Corolario 3.3.1. Sean $E$ un cogenerador inyectivo de $A$ y $M$ un A-módulo E-Matlis reflexivo, entonces existe un isomorfismo natural

$$
\mathrm{H}_{0}^{\mathfrak{a}, \varphi}(M) \cong \lim _{\mathfrak{b} \in \overleftarrow{\mathcal{G}(\mathfrak{a}, \varphi)}} M / \mathfrak{b} M
$$

Prueba. Basta recordar que

$$
\operatorname{Tor}_{0}^{A}(A / \mathfrak{b}, M) \cong A / \mathfrak{b} \otimes_{A} M \cong M / \mathfrak{b} M
$$

y aplicar el Teorema 3.3.1.

Corolario 3.3.2. Sean $E$ un cogenerador inyectivo de $A$ y $M$ un A-módulo E-Matlis reflexivo, entonces

$$
\mathrm{H}_{i}^{\mathfrak{a}, \varphi}\left(D_{E} M\right) \cong D_{E}\left(\mathrm{H}_{\mathfrak{a}, \varphi}^{i}(M)\right) .
$$

Prueba. Por la Proposición 3.3.1, $D_{E} M$ es un $A$-módulo E-Matlis reflexivo. Luego, por el Teorema 3.3.1, el Lema 3.3.1 y el Corolario 2.3.1, obtenemos lo siguiente.

$$
\begin{aligned}
\mathrm{H}_{i}^{\mathfrak{a}, \varphi}\left(D_{E} M\right) & \cong \lim _{\mathfrak{b} \in \overleftarrow{\mathcal{G}(\mathfrak{a}, \varphi)}} \operatorname{Tor}_{i}^{A}\left(A / \mathfrak{b}, D_{E} M\right) \\
& \cong \lim _{\mathfrak{b} \in \overleftarrow{\mathcal{G}(\mathfrak{a}, \varphi)}} D_{E}\left(\operatorname{Ext}_{A}^{i}(A / \mathfrak{b}, M)\right) \\
& \cong D_{E}\left(\underset{\mathfrak{b} \in \overrightarrow{\mathcal{G}(\mathfrak{a}, \varphi)}}{\lim _{A}} \operatorname{Ext}_{A}^{i}(A / \mathfrak{b}, M)\right) \\
& \cong D_{E}\left(\mathrm{H}_{\mathfrak{a}, \varphi}^{i}(M)\right)
\end{aligned}
$$

Corolario 3.3.3. Sea $0 \longrightarrow L \longrightarrow M \longrightarrow N \longrightarrow 0$ una sucesión exacta corta de módulos Artinianos, entonces existe una sucesión exacta larga en los módulos de homología local con respecto al par $(\mathfrak{a}, \varphi)$

$$
\cdots \longrightarrow \mathrm{H}_{1}^{\mathfrak{a}, \varphi}(M) \longrightarrow \mathrm{H}_{1}^{\mathfrak{a}, \varphi}(N) \longrightarrow \mathrm{H}_{0}^{\mathfrak{a}, \varphi}(L) \longrightarrow \mathrm{H}_{0}^{\mathfrak{a}, \varphi}(M) \longrightarrow \mathrm{H}_{0}^{\mathfrak{a}, \varphi}(N) \longrightarrow 0 .
$$


Prueba. Para cada $\mathfrak{b} \in \mathcal{G}(\mathfrak{a}, \varphi)$, la sucesión exacta corta $0 \longrightarrow L \longrightarrow M \longrightarrow N \longrightarrow 0$ induce una sucesión exacta larga

$$
\begin{gathered}
\cdots \longrightarrow \operatorname{Tor}_{i}^{A}(A / \mathfrak{b}, L) \longrightarrow \operatorname{Tor}_{i}^{A}(A / \mathfrak{b}, M) \longrightarrow \operatorname{Tor}_{i}^{A}(A / \mathfrak{b}, N) \longrightarrow \cdots \\
\cdots \rightarrow A / \mathfrak{b} \otimes L \longrightarrow A / \mathfrak{b} \otimes M \longrightarrow A / \mathfrak{b} \otimes N \longrightarrow 0 .
\end{gathered}
$$

Desde que $L, M$ y $N$ son $A$-módulos Artinianos, los módulos que aparecen en la sucesión exacta larga también son Artinianos. Por [7, Corollaire 7.2] el funtor límite inverso es exacto en la categoría de los $A$-módulos Artinianos. Por lo tanto, aplicando $\underset{\mathfrak{b} \in \overleftarrow{\mathcal{G}(\mathfrak{a}, \varphi)}}{\lim }(-)$, obtenemos la sucesión exacta larga

$$
\begin{gathered}
\cdots \longrightarrow \lim _{\mathfrak{b} \in \overleftarrow{\mathcal{G}(\mathfrak{a}, \varphi)}} \operatorname{Tor}_{i}^{A}(A / \mathfrak{b}, L) \longrightarrow \lim _{\mathfrak{b} \in \overleftarrow{\mathcal{G}(\mathfrak{a}, \varphi)}} \operatorname{Tor}_{i}^{A}(A / \mathfrak{b}, M) \longrightarrow \lim _{\mathfrak{b} \in \overleftarrow{\mathcal{G}(\mathfrak{a}, \varphi)}} \operatorname{Tor}_{i}^{A}(A / \mathfrak{b}, N) \longrightarrow \cdots \\
\quad \cdots \rightarrow \lim _{\mathfrak{b} \in \overleftarrow{\mathcal{G}(\mathfrak{a}, \varphi)}} A / \mathfrak{b} \otimes L \longrightarrow \underbrace{}_{\mathfrak{b} \in \overleftarrow{\mathcal{G}(\mathfrak{a}, \varphi)}} A / \mathfrak{b} \otimes M \longrightarrow \lim _{\mathfrak{b} \in \overleftarrow{\mathcal{G}(\mathfrak{a}, \varphi)}} A / \mathfrak{b} \otimes N \longrightarrow 0 .
\end{gathered}
$$

Finalmente, como módulos Artinianos son Matlis reflexivos, el Teorema 3.3.1 demuestra que la última sucesión es precisamente la sucesión exacta larga deseada.

\section{Conclusión}

- Los funtores de cohomología local introducidos en este trabajo generalizan aquellos introducidos por R. Takahashi, Y. Yoshini and Y. Yoshisawa en [9]. A diferencia de los autores citados, nuestra teoría es estudiada en el contexto de la cohomología local expuesta en la Sección 2 ,

- Dentro de nuestros resultados obtenidos resaltamos el Teorema 3.2.1, que permite calcular la cohomología local en términos de la cohomología del complejo generalizado de Čech; y el Teorema 3.3.1, que expresa la homología local de un módulo Matlis reflexivo como un límite inverso de ciertos módulos Tor.

- El Corolario 3.3.2 establece una dualidad entre las nociones de cohomología y homología local.

\section{Referencias bibliográficas}

[1] L. A. Alba-Sarria, R. Callejas-Bedregal, and N. Caro-Tuesta (2020). A local cohomology theory defined by a system of ideals . Preprint.

[2] R. G. Belshoff, E. E. Enochs, and J. R. García Rozas (2000). Generalized Matlis Duality. Proc. Amer. Math. Soc., 128:1307-1312.

[3] T. Y. Brodmann and R. Sharp (2013). Local Cohomology: An algebraic introduction with geometric applications. Cambridge Studies in Advanced Mathematics, Cambridge University Press.

[4] K. Divanni-Azar, R. Naghipour and M. Tousi (2002). The Lichtenbaum-Hartshorne theorem for generalized local cohomology and connectnedss. Comm., 30(8):3687-3702.

[5] A. Grothendieck. (2005). Séminaire de Géométrie Algébrique du Bois Marie - 1962 - Cohomologie locale des faisceaux cohérents et théorèmes de Lefschetz locaux et globaux - (SGA 2). Société Mathématique de France. 
[6] R. Hartshorne (1969). A seminar given by A. Grothendieck, Harvard University, Fall, 1961. Lecture Notes in Mathematics. Springer Verlag, Berlin, New York.

[7] C. Jensen (1972). Les foncteurs Dérivés de lim et leurs Applications en Théorie des Modules. Springer-Verlag, Berlin/Heidelberg/New York.

[8] J. J. Rotman (2009). An Introduction to Homological Algebra. Universitext, Springer-Verlag.

[9] R. Takahashi, Y. Yoshino, and T. Yoshizawa (2009). Local cohomology based on nonclosed support defined by a pair of ideals. J. Pure Appl. Algebra, (4):582-600. 